\title{
Symmetric tensor fields of bounded deformation
}

\author{
Kristian Bredies
}

Received: 19 July 2011 / Accepted: 27 December 2011 / Published online: 12 January 2012

(C) Fondazione Annali di Matematica Pura ed Applicata and Springer-Verlag 2012

\begin{abstract}
We introduce and study spaces of symmetric tensor fields of bounded deformation for tensors of arbitrary order, i.e., where the symmetrized derivative is still a Radon measure. A Sobolev-Korn type estimate, a boundary trace theorem and continuous as well as compact embedding properties into Lebesgue spaces are obtained, showing that these spaces can be regarded as a natural generalization of the spaces of bounded deformation to higher-order symmetric tensors.
\end{abstract}

Keywords Symmetric tensor fields · Bounded deformation - Sobolev-Korn inequality . Boundary traces · Continuous/compact embeddings

Mathematics Subject Classification (2000) $\quad 46 \mathrm{E} 35 \cdot 53 \mathrm{~A} 45 \cdot 74 \mathrm{~B} 20$

\section{Introduction}

This article is concerned with the study of spaces of symmetric tensor fields which we call of bounded deformation. The main feature of elements of these spaces is that their symmetrized weak derivatives are still tensor-valued Radon measures:

$$
\begin{aligned}
\operatorname{BD}\left(\Omega, \operatorname{Sym}^{k}\left(\mathbb{R}^{d}\right)\right) & =\left\{u \in L^{1}\left(\Omega, \operatorname{Sym}^{k}\left(\mathbb{R}^{d}\right)\right) \mid \mathcal{E}(u) \in \mathcal{M}\left(\Omega, \operatorname{Sym}^{k+1}\left(\mathbb{R}^{d}\right)\right)\right\} \\
\|u\|_{\mathrm{BD}} & =\|u\|_{1}+\|\mathcal{E}(u)\|_{\mathcal{M}} .
\end{aligned}
$$

As Korn's inequality is known not to hold in the measure or $L^{1}$ - case, this concept is weaker than the notion of bounded variation. In fact, for vector fields, the spaces correspond to

Support by the special research grant SFB "Mathematical Optimization and Applications in Biomedical Sciences" of the Austrian Science Fund (FWF) is gratefully acknowledged.

K. Bredies $(\bowtie)$

Institute of Mathematics and Scientific Computing, University of Graz,

Heinrichstrasse 36, 8010 Graz, Austria

e-mail: kristian.bredies@uni-graz.at 
the well-known spaces of bounded deformation [16], which admit, although weaker, many convenient functional-analytical properties like (compact) Sobolev embeddings, integrable traces and the validity of the divergence theorem. For tensors of higher orders, the spaces of bounded deformation as well as the above-mentioned properties are, to the best knowledge of the author, not known in the literature. The main contribution of this paper is therefore the generalization of these notions and results to the case of symmetric tensor fields of arbitrary order.

The introduction of spaces of vector fields of bounded deformation can be motivated, on the one hand, in the context of the mathematical theory of plasticity. In infinitesimal strain theory, the strain is expressed by the symmetrized gradient of the displacement field. One common mathematical modeling approach relates strain to stress by some constitutive law which is usually expressed as a pointwise subgradient inclusion. The displacement fields for a static configuration are therefore characterized by the solutions of minimization problems involving some strain energy functional. In particular, for energy functionals that are related to (perfect) plasticity (such as the Hencky plasticity model), solutions can only be proven to exist for vector fields of bounded deformation $[2,15]$. Furthermore, the space of vector fields of (special) bounded deformation is believed to be an appropriate solution set for problems related to brittle fracture [9]. Recent developments indicate that for modeling special materials, higher-order terms may be incorporated $[6,14]$ leading to tensor fields of higher order which are often symmetric.

A different context in which symmetric tensor fields of bounded deformation appear naturally is in the study of the total generalized variation (TGV) functional introduced in [4]: Using Fenchel-Rockafellar duality, $\mathrm{TGV}_{\alpha}^{k}$ of a locally integrable function $u: \Omega \rightarrow \mathbb{R}$ can informally be written as

$$
\operatorname{TGV}_{\alpha}^{k}(u)=\inf _{\substack{u_{l} \in \operatorname{BD}\left(\Omega, \operatorname{Sym}^{l}\left(\mathbb{R}^{d}\right)\right) \\ l=0, \ldots, k, u_{0}=u, u_{k}=0}} \sum_{l=1}^{k} \alpha_{k-l}\left\|\mathcal{E}\left(u_{l-1}\right)-u_{l}\right\|_{\mathcal{M}}
$$

For the case $k=2$, it has been shown in [5] that this representation leads to $\mathrm{TGV}_{\alpha}^{2}$ being embedded into the common $L^{p}$-framework: In particular, regularization properties for a large class of ill-posed inverse problems could be established. These results, however, strongly rely on the embedding properties of $\operatorname{BD}\left(\Omega, \operatorname{Sym}^{1}\left(\mathbb{R}^{d}\right)\right)$ into $L^{p}$-spaces as well as the validity of a Sobolev-Korn estimate. In order to generalize the results, the corresponding versions of these results have to be available for $\operatorname{BD}\left(\Omega, \operatorname{Sym}^{k}\left(\mathbb{R}^{d}\right)\right)$ with arbitrary $k$.

Motivated by the above, this article deals with the functional analytic background of such tensor fields by studying spaces of non-smooth fields that we call, analogously, symmetric tensor fields of bounded deformation. The main results can roughly be seen to be the analogous versions of what is known for functions of bounded variation, see the next paragraph for a more detailed discussion. It should be emphazised, however, that the adaptation of these statements to the general tensor-field case is far from being trivial: One has to deal with the fact that the algebraic structure of symmetric tensor fields becomes more complex with growing order. The kernel of the symmetrized derivative $\mathcal{E}$ and the fundamental solutions of $\operatorname{div} \mathcal{E}$ for symmetric tensor fields of order $k$ are therefore, except for the known cases $k \in\{0,1\}$, not immediate, and dedicated considerations involving, for example, algebraic graph theory have to be carried out to obtain respective representations.

The outline of the paper is as follows. First, in Sect. 2, the necessary prerequisites for the introduction of symmetric tensor fields of bounded deformation are introduced. In Sect. 3, we deduce some basic properties about the symmetrized derivative, in particular, we determine 
its kernel, the infinitesimal rigid displacements, and derive the fundamental solutions of the corresponding "linear elasticity equations". Also, a proof of Korn's inequality in a basic situation can be found. These results play a crucial role later on in Sect. 4 which contains the main results:

1. We derive a Sobolev-Korn type estimate for smooth, compactly supported tensor fields of bounded deformation as follows:

$$
\|u\|_{d /(d-1)} \leq C\|\mathcal{E}(u)\|_{1},
$$

see Theorem 4.8 .

2. For bounded domains with Lipschitz boundary, a trace theorem is established showing that tensor fields of bounded deformation admit an integrable trace on the boundary in terms of

$$
\|u\|_{1, \partial \Omega} \leq C\|u\|_{\mathrm{BD}},
$$

see Theorem 4.12. Moreover, the validity of the "integration by parts" formula for tensor fields of bounded deformation is verified (Theorem 4.14).

3. Embedding theorems for the space of tensor fields of bounded deformation on a bounded Lipschitz domain $\Omega$ are proven, specifically,

$$
\mathrm{BD}\left(\Omega, \operatorname{Sym}^{k}\left(\mathbb{R}^{d}\right)\right) \hookrightarrow L^{p}\left(\Omega, \operatorname{Sym}^{k}\left(\mathbb{R}^{d}\right)\right)
$$

for $1 \leq p \leq d /(d-1)$, see Theorem 4.16. These embeddings are compact when the exponent is below the critical value $d /(d-1)$ (Theorem 4.17).

4. Finally, a Sobolev-Korn type inequality of the type

$$
\|u-R u\|_{p} \leq C\|\mathcal{E}(u)\|_{\mathcal{M}}
$$

with $p \in[1, d /(d-1)]$ and $R$ being a projection on the infinitesimal rigid displacements of order $k$ is established (Theorem 4.19 and Corollary 4.20).

\section{Preliminaries}

In order to introduce spaces of integrable and smooth symmetric tensor fields, let us first fix some notation concerning tensors. It is also useful to recall some known facts from tensor analysis which will be utilized later on.

Throughout the paper, $d \geq 1$ denotes the space dimension. Let

$$
\begin{aligned}
\mathcal{T}^{k}\left(\mathbb{R}^{d}\right) & =\{\xi: \underbrace{\mathbb{R}^{d} \times \cdots \times \mathbb{R}^{d}}_{k \text {-times }} \rightarrow \mathbb{R} \mid \xi k \text {-linear }\}, \\
\operatorname{Sym}^{k}\left(\mathbb{R}^{d}\right) & =\{\xi: \underbrace{\mathbb{R}^{d} \times \cdots \times \mathbb{R}^{d}}_{k \text {-times }} \rightarrow \mathbb{R} \mid \xi k \text {-linear and symmetric }\}
\end{aligned}
$$

be the vector space of $k$-tensors and symmetric $k$-tensors, respectively (actually, these are spaces of $(0, k)$-tensors, but since we only deal with covariant tensors, we omit the 0$)$. Obviously, the latter is a subspace of the former. With $\operatorname{Sym}^{k}\left(\mathbb{R}^{d}\right) \otimes \operatorname{Sym}^{l}\left(\mathbb{R}^{d}\right)$, we denote the subspace of $\mathcal{T}^{k+l}\left(\mathbb{R}^{d}\right)$ consisting of $(k+l)$-tensors which are symmetric in the first $k$ variables and in the last $l$-variables. 
Note three basic operations for $k$-tensors. For $\xi \in \mathcal{T}^{k}\left(\mathbb{R}^{d}\right)$ and $\eta \in \mathcal{T}^{l}\left(\mathbb{R}^{d}\right)$, the tensor product

$$
(\xi \otimes \eta)\left(a_{1}, \ldots, a_{k+l}\right)=\xi\left(a_{1}, \ldots, a_{k}\right) \eta\left(a_{k+1}, \ldots, a_{k+l}\right)
$$

yields an element of $\mathcal{T}^{k+l}\left(\mathbb{R}^{d}\right)$. The trace of a $\xi \in \mathcal{T}^{k}\left(\mathbb{R}^{d}\right)$ with $k \geq 2$ is defined by the contraction

$$
\operatorname{tr}(\xi)\left(a_{1}, \ldots, a_{k-2}\right)=\sum_{i=1}^{d} \xi\left(e_{i}, a_{1}, \ldots, a_{k-2}, e_{i}\right)
$$

where $e_{i}$ denotes the $i$ th standard basis vector in $\mathbb{R}^{d}$. This operation can be iterated, for example, $\operatorname{tr}^{l}(\xi \otimes \eta)$ for $\xi \in \operatorname{Sym}^{k+l}\left(\mathbb{R}^{d}\right)$ and $\eta \in \operatorname{Sym}^{l}\left(\mathbb{R}^{d}\right)$ yields a symmetric $k$-tensor. Every $k$-tensor $\xi \in \mathcal{T}^{k}\left(\mathbb{R}^{d}\right)$ can be symmetrized by

$$
(\| \mid \xi)\left(a_{1}, \ldots, a_{k}\right)=\frac{1}{k !} \sum_{\pi \in S_{k}} \xi\left(a_{\pi(1)}, \ldots, a_{\pi(k)}\right)
$$

where $S_{k}$ denotes the permutation group of $\{1, \ldots, k\}$.

The spaces $\mathcal{T}^{k}\left(\mathbb{R}^{d}\right)$ and, consequently, $\operatorname{Sym}^{k}\left(\mathbb{R}^{d}\right)$ will be equipped with the scalar product

$$
\begin{aligned}
\xi \cdot \eta=\operatorname{tr}^{k}(\xi \otimes \bar{\eta}) & =\sum_{p \in\{1, \ldots, d\}^{k}} \xi\left(e_{p_{1}}, \ldots, e_{p_{k}}\right) \eta\left(e_{p_{1}}, \ldots, e_{p_{k}}\right), \\
\bar{\eta}\left(a_{1}, \ldots, a_{k}\right) & =\eta\left(a_{k}, \ldots, a_{1}\right)
\end{aligned}
$$

leading canonically to the norm $|\xi|=\sqrt{\xi \cdot \xi}$. This norm is compatible with the Euclidean norm in $\mathbb{R}^{d}$ in the sense that $\left|\xi\left(a_{1}, \ldots, a_{k}\right)\right| \leq|\xi|\left|a_{1}\right| \cdots\left|a_{k}\right|$. The scalar product moreover possesses the property that the symmetrization of a $k$-tensor becomes the orthogonal projection onto $\operatorname{Sym}^{k}\left(\mathbb{R}^{d}\right)$. Indeed, for all $\xi \in \mathcal{T}^{k}\left(\mathbb{R}^{d}\right)$ and $\eta \in \operatorname{Sym}^{k}\left(\mathbb{R}^{d}\right)$, we have

$$
\begin{aligned}
(\| \xi) \cdot \eta & =\frac{1}{k !} \sum_{\pi \in S_{k}} \sum_{p \in\{1, \ldots, d\}^{k}} \xi\left(e_{p_{\pi(1)}}, \ldots, e_{p_{\pi(k)}}\right) \eta\left(e_{p_{1}}, \ldots, e_{p_{k}}\right) \\
& =\frac{1}{k !} \sum_{p \in\{1, \ldots, d\}^{k}} \xi\left(e_{p_{1}}, \ldots, e_{p_{k}}\right) \sum_{\pi \in S_{k}} \eta\left(e_{p_{\pi(1)}}, \ldots, e_{p_{\pi(k)}}\right)=\xi \cdot \eta .
\end{aligned}
$$

In order to describe the structure of $\operatorname{Sym}^{k}\left(\mathbb{R}^{d}\right)$ as a vector space, it is useful to consider the relation of mappings $p \in\{1, \ldots, d\}^{k}$ to multiindices $\beta \in \mathbb{N}^{d}$ with $|\beta|=k$ :

$$
\sigma:\{1, \ldots, d\}^{k} \rightarrow \mathbb{N}^{d} \quad, \quad \sigma(p)_{i}=\left|\left\{k \mid p_{k}=i\right\}\right| .
$$

For each $\beta \in \mathbb{N}^{d}$ with $|\beta|=k$, one can associate a $p \in\{1, \ldots, d\}^{k}$ by

$$
\sigma^{-1}(\beta)_{j}=\min \left\{m \mid \sum_{n=1}^{m} \beta_{n} \geq j\right\}
$$

which is only a right inverse of $\sigma$. In fact, there are several $p$ for which $\sigma(p)=\beta$, and its cardinality is known to be

$$
|\{p \mid \sigma(p)=\beta\}|=\frac{k !}{\beta !}=\frac{k !}{\beta_{1} ! \cdots \beta_{d} !} .
$$


The multiindex notation reflects the fact that the order of elements does not matter for the symmetry we are considering. It is known that a basis of $\operatorname{Sym}^{k}\left(\mathbb{R}^{d}\right)$ can be obtained by

$$
\beta \in \mathbb{N}^{d},|\beta|=k: \quad e_{\beta}\left(a_{1}, \ldots, a_{k}\right)=\sum_{\substack{p \in\{1, \ldots, d\}^{k} \\ \sigma(p)=\beta}} \prod_{j=1}^{k} a_{j, p_{j}}
$$

for which the coefficients are given by $\xi_{\beta}=\xi\left(e_{p_{1}}, \ldots, e_{p_{k}}\right)$ where $p=\sigma^{-1}(\beta)$. Counting multiplicities, we can express

$$
(\xi \otimes \eta)_{\beta, \gamma}=\xi_{\beta} \eta_{\gamma} \quad, \quad \operatorname{tr}^{l}(\xi \otimes \eta)_{\beta}=\sum_{p \in\{1, \ldots, d\}^{l}} \xi_{\beta+\sigma(p)} \eta_{\sigma(p)}=\sum_{|\gamma|=l} \frac{l !}{\gamma !} \xi_{\beta+\gamma} \eta_{\gamma}
$$

in particular, the scalar product on $\operatorname{Sym}^{k}\left(\mathbb{R}^{d}\right)$ is $\xi \cdot \eta=\sum_{|\beta|=k} \frac{k !}{\beta !} \xi_{\beta} \eta_{\beta}$. Having the basis $\left\{e_{\beta}\right\}$, the equivalence between $\operatorname{Sym}^{k}\left(\mathbb{R}^{d}\right)$ and the space of homogenous polynomials of degree $k$ becomes clear: For each $\xi \in \operatorname{Sym}^{k}\left(\mathbb{R}^{d}\right)$, we can associate

$$
p: \mathbb{R}^{d} \rightarrow \mathbb{R}: p_{\xi}(x)=\xi(x, \ldots, x)=\sum_{|\beta|=k} \xi_{\beta} x^{\beta} \quad, \quad \frac{\partial^{k} p_{\xi}}{\partial x^{\beta}}(0)=\beta ! \xi_{\beta},
$$

the latter showing that the relation is one-to-one.

Next, let $\Omega \subset \mathbb{R}^{d}$ be a fixed domain. We define symmetric $k$-tensor fields as mappings $\xi: \Omega \rightarrow \operatorname{Sym}^{k}\left(\mathbb{R}^{d}\right)$ and associate Lebesgue spaces with them.

$$
\begin{array}{r}
L^{p}\left(\Omega, \operatorname{Sym}^{k}\left(\mathbb{R}^{d}\right)\right)=\left\{\xi: \Omega \rightarrow \operatorname{Sym}^{k}\left(\mathbb{R}^{d}\right) \text { measurable } \mid\|\xi\|_{p}<\infty\right\} \\
\|\xi\|_{p}=\left(\int_{\Omega}|\xi(x)|^{p} \mathrm{~d} x\right)^{1 / p} \text { for } 1 \leq p<\infty \quad, \quad\|\xi\|_{\infty}=\underset{x \in \Omega}{\operatorname{ess} \sup }|\xi(x)| .
\end{array}
$$

Let the spaces $L_{\text {loc }}^{p}\left(\Omega, \operatorname{Sym}^{k}\left(\mathbb{R}^{d}\right)\right)$ be defined through the usual modification. It should be noted that, since the vector norm in $\operatorname{Sym}^{k}\left(\mathbb{R}^{d}\right)$ is a scalar product, the usual duality holds: $L^{p}\left(\Omega, \operatorname{Sym}^{k}\left(\mathbb{R}^{d}\right)\right)^{*}=L^{p^{*}}\left(\Omega, \operatorname{Sym}^{k}\left(\mathbb{R}^{d}\right)\right)$ for $1 \leq p<\infty$ and $1 / p+1 / p^{*}=1$.

Moreover, denote by $\mathcal{C}\left(\bar{\Omega}, \operatorname{Sym}^{k}\left(\mathbb{R}^{d}\right)\right)$ the usual space of continuous functions as well as

$$
\begin{aligned}
\mathcal{C}_{\mathrm{c}}\left(\Omega, \operatorname{Sym}^{k}\left(\mathbb{R}^{d}\right)\right)= & \left\{\xi \in \mathcal{C}\left(\bar{\Omega}, \operatorname{Sym}^{k}\left(\mathbb{R}^{d}\right)\right) \mid \operatorname{supp} \xi \subset \subset \Omega\right\}, \\
& \mathcal{C}_{0}\left(\Omega, \operatorname{Sym}^{k}\left(\mathbb{R}^{d}\right)\right)=\overline{\mathcal{C}_{\mathrm{c}}\left(\Omega, \operatorname{Sym}^{k}\left(\mathbb{R}^{d}\right)\right) .}
\end{aligned}
$$

Again, duality holds as expected: $\mathcal{C}_{0}\left(\Omega, \operatorname{Sym}^{k}\left(\mathbb{R}^{d}\right)\right)^{*}=\mathcal{M}\left(\Omega, \operatorname{Sym}^{k}\left(\mathbb{R}^{d}\right)\right)$ the latter being the space of $\operatorname{Sym}^{k}\left(\mathbb{R}^{d}\right)$-valued "signed" Radon measures. Such a measure $\mu$ can be decomposed into the total variation measure $|\mu|$ and a $|\mu|$-measurable $\tau: \Omega \rightarrow \operatorname{Sym}^{k}\left(\mathbb{R}^{d}\right)$ with $|\tau(x)|=1|\mu|$-almost everywhere. The duality relation then becomes

$$
\langle\mu, \xi\rangle_{\mathcal{M} \times \mathcal{C}_{0}}=\int_{\Omega} \xi(x) \cdot \tau(x) \mathrm{d}|\mu|(x) \quad \text { for all } \xi \in \mathcal{C}_{0}\left(\Omega, \operatorname{Sym}^{k}\left(\mathbb{R}^{d}\right)\right) .
$$

Let us now discuss differentiation. The derivative of a symmetric $k$-tensor field is in general a non-symmetric tensor field, and we use the notation

$$
(\nabla \otimes \xi)(x)\left(a_{1}, \ldots, a_{k+1}\right)=\left(\mathrm{D} \xi(x)\left(a_{1}\right)\right)\left(a_{2}, \ldots, a_{k+1}\right),
$$


as well as

$$
(\xi \otimes \nabla)(x)\left(a_{1}, \ldots, a_{k+1}\right)=\left(\mathrm{D} \xi(x)\left(a_{k+1}\right)\right)\left(a_{1}, \ldots, a_{k}\right) .
$$

We are in particular interested in the symmetrized derivative

$$
\mathcal{E}(\xi)=\|(\nabla \otimes \xi)=\|(\xi \otimes \nabla) .
$$

Spaces of continuously differentiable functions in this sense are defined as:

$$
\begin{aligned}
\mathcal{C}^{1}\left(\bar{\Omega}, \operatorname{Sym}^{k}\left(\mathbb{R}^{d}\right)\right) & =\left\{\xi: \bar{\Omega} \rightarrow \operatorname{Sym}^{k}\left(\mathbb{R}^{d}\right) \mid \nabla \otimes \xi \text { continuous on } \bar{\Omega}\right\} \\
\|\xi\|_{1, \infty} & =\max \left\{\|\xi\|_{\infty},\|\nabla \otimes \xi\|_{\infty}\right\}
\end{aligned}
$$

We also like to use differentiable symmetric $k$-tensor fields with compact support in $\Omega$ :

$$
\mathcal{C}_{\mathrm{c}}^{1}\left(\Omega, \operatorname{Sym}^{k}\left(\mathbb{R}^{d}\right)\right)=\left\{\xi \in \mathcal{C}^{1}\left(\bar{\Omega}, \operatorname{Sym}^{k}\left(\mathbb{R}^{d}\right)\right) \mid \operatorname{supp} \xi \subset \subset \Omega\right\} .
$$

In order to introduce weak derivatives, one needs the notion of divergence of a symmetric $(k+1)$-tensor field:

$$
(\operatorname{div} \xi)=\operatorname{tr}(\nabla \otimes \xi)=\operatorname{tr}(\xi \otimes \nabla) \quad, \quad(\operatorname{div} \xi)_{\beta}=\sum_{i=1}^{d} \frac{\partial \xi_{\beta+e_{i}}}{\partial x_{i}}
$$

It should be noted that $\operatorname{div} \xi$ is a symmetric $k$-tensor field. With the definition of the divergence according to (2.1), the validity of a corresponding integration by parts formula can be verified.

Proposition 2.1 Let $\Omega$ be a bounded Lipschitz domain and $\xi \in \mathcal{C}^{1}\left(\bar{\Omega}, \operatorname{Sym}^{k+1}\left(\mathbb{R}^{d}\right)\right)$ be a smooth symmetric $(k+1)$-tensor field. Then, for each smooth symmetric $k$-tensor field $\eta \in \mathcal{C}^{1}\left(\bar{\Omega}, \operatorname{Sym}^{k}\left(\mathbb{R}^{d}\right)\right)$ we have:

$$
\int_{\Omega} \operatorname{div} \xi \cdot \eta \mathrm{d} x=\int_{\partial \Omega} \xi \cdot \|(\eta \otimes v) \mathrm{d} \mathcal{H}^{d-1}-\int_{\Omega} \xi \cdot \mathcal{E}(\eta) \mathrm{d} x
$$

where $v$ denotes the outward unit normal on $\partial \Omega$.

Proof This statement follows from the usual integration by parts formula. Observe that $p \in\{1, \ldots, d\}^{k}$ and $i=1, \ldots, d$ gives each $(p, i) \in\{1, \ldots, d\}^{k+1}$, and we can express $\sigma((p, i))=\sigma(p)+e_{i}$. Therefore, with integration by parts and using that the symmetrization 
is the orthogonal projection onto the space of symmetric $k$-tensors,

$$
\begin{aligned}
\int_{\Omega} \operatorname{div} \xi \cdot \eta \mathrm{d} x= & \int_{\Omega} \operatorname{tr}^{k}(\operatorname{tr}(\nabla \otimes \xi) \otimes \eta) \mathrm{d} x \\
= & \int_{\Omega} \sum_{(p, i) \in\{1, \ldots, d\}^{k+1}} \frac{\partial \xi_{\sigma(p)+e_{i}}}{\partial x_{i}} \eta_{\sigma(p)} \mathrm{d} x \\
= & \int_{\partial \Omega} \sum_{(p, i) \in\{1, \ldots, d\}^{k+1}} \xi_{\sigma(p)+e_{i}} v_{i} \eta_{\sigma(p)} \mathrm{d} \mathcal{H}^{d-1} \\
& -\int_{\Omega} \sum_{(p, i) \in\{1, \ldots, d\}^{k+1}} \xi_{\sigma((p, i))} \frac{\partial \eta_{\sigma(p)}}{\partial x_{i}} \mathrm{~d} x \\
= & \int_{\partial \Omega} \xi \cdot(\eta \otimes v) \mathrm{d} \mathcal{H}^{d-1}-\int_{\Omega} \xi \cdot(\nabla \otimes \eta) \mathrm{d} x \\
= & \int_{\partial \Omega} \xi \cdot \|(\eta \otimes v) \mathrm{d} \mathcal{H}^{d-1}-\int_{\Omega} \xi \cdot \mathcal{E}(\eta) \mathrm{d} x
\end{aligned}
$$

yielding the desired identity.

Remark 2.2 It is easy to see that if $\xi$ has compact support in $\Omega$, then (2.2) holds with the boundary term being zero and for arbitrary domains.

Remark 2.3 If one defines $\operatorname{div} \xi=\operatorname{tr}(\xi \otimes \nabla)$ for $\xi \in \mathcal{C}^{1}\left(\bar{\Omega}, \mathcal{T}^{k+1}\left(\mathbb{R}^{d}\right)\right)$, an analogous identity holds for $\eta \in \mathcal{C}^{1}\left(\bar{\Omega}, \mathcal{T}^{k}\left(\mathbb{R}^{d}\right)\right)$ :

$$
\int_{\Omega} \operatorname{div} \xi \cdot \eta \mathrm{d} x=\int_{\partial \Omega} \xi \cdot(v \otimes \eta) \mathrm{d} \mathcal{H}^{d-1}-\int_{\Omega} \xi \cdot(\nabla \otimes \eta) \mathrm{d} x .
$$

Based on the identities (2.2) and (2.3), we define weak derivatives of symmetric $k$-tensor fields.

Definition 2.4 For $\xi \in L_{\text {loc }}^{1}\left(\Omega, \mathcal{T}^{k}\left(\mathbb{R}^{d}\right)\right)$, an $\eta \in L_{\text {loc }}^{1}\left(\Omega, \mathcal{T}^{k+1}\left(\mathbb{R}^{d}\right)\right)$ is called the weak derivative of $\xi$ if the identity

$$
\int_{\Omega} \eta \cdot \zeta \mathrm{d} x=-\int_{\Omega} \xi \cdot \operatorname{div} \zeta \mathrm{d} x
$$

is satisfied for all $\zeta \in \mathcal{C}_{\mathrm{c}}^{1}\left(\Omega, \mathcal{T}^{k+1}\left(\mathbb{R}^{d}\right)\right)$. We also write $\eta=\nabla \otimes \xi$ in this case.

Likewise, $\eta \in L_{\text {loc }}^{1}\left(\Omega, \operatorname{Sym}^{k+1}\left(\mathbb{R}^{d}\right)\right)$ is called the weak symmetrized derivative of $\xi \in$ $L_{\text {loc }}^{1}\left(\Omega, \operatorname{Sym}^{k}\left(\mathbb{R}^{d}\right)\right)$ if the identity

$$
\int_{\Omega} \eta \cdot \zeta \mathrm{d} x=-\int_{\Omega} \xi \cdot \operatorname{div} \zeta \mathrm{d} x
$$

is satisfied for all $\zeta \in \mathcal{C}_{\mathrm{c}}^{1}\left(\Omega, \operatorname{Sym}^{k+1}\left(\mathbb{R}^{d}\right)\right)$. In this case, we denote $\mathcal{E}(\xi)=\eta$.

Note again that in the latter case, since we only test with symmetric $(k+1)$-tensor fields, we are indeed only able to determine the symmetric part of the gradient. The Sobolev spaces 
associated with such a notion of derivative are then given by:

$$
\begin{aligned}
H^{1, p}\left(\Omega, \operatorname{Sym}^{k}\left(\mathbb{R}^{d}\right)\right) & =\left\{\xi \in L^{p}\left(\Omega, \operatorname{Sym}^{k}\left(\mathbb{R}^{d}\right)\right) \mid \mathcal{E}(\xi) \in L^{p}\left(\Omega, \operatorname{Sym}^{k+1}\left(\mathbb{R}^{d}\right)\right)\right\}, \\
\|\xi\|_{1, p} & =\left(\|\xi\|_{p}^{p}+\|\mathcal{E}(\xi)\|_{p}^{p}\right)^{1 / p} \text { for } 1 \leq p<\infty \\
\|\xi\|_{1, \infty} & =\max \left\{\|\xi\|_{\infty},\|\mathcal{E}(\xi)\|_{\infty}\right\} .
\end{aligned}
$$

For the vector field case $k=1$, many variants of Korn's inequality are known, essentially stating that for $\xi \in L^{p}\left(\Omega, \operatorname{Sym}^{1}\left(\mathbb{R}^{d}\right)\right)$ the equivalence

$$
\mathcal{E}(\xi) \in L^{p}\left(\Omega, \operatorname{Sym}^{2}\left(\mathbb{R}^{d}\right)\right) \Leftrightarrow \nabla \otimes \xi \in L^{p}\left(\Omega, \mathcal{T}^{2}\left(\mathbb{R}^{d}\right)\right)
$$

holds if $1<p<\infty$ and $\Omega$ is sufficiently smooth [11]. It is therefore not necessary to distinguish between $H^{1, p}\left(\Omega, \operatorname{Sym}^{1}\left(\mathbb{R}^{d}\right)\right)$ defined above and the usual Sobolev space $H^{1, p}\left(\Omega, \mathbb{R}^{d}\right)$. For arbitrary $k$, a Korn inequality holds for $p=2$ (see Proposition 3.6) suggesting that the situation is similar for higher-order tensors. We will, however, not address this as the main objective of this paper was to study the situation where Korn's inequality does not hold as it is the case for vector fields of bounded deformation and, as we will see, also not for tensor fields.

\section{The symmetrized derivative}

This section is devoted to deriving some facts for the symmetrized derivative of tensor fields of any order which seem not to appear in the existing literature (to the best knowledge of the author). We are, on the one hand, interested in describing its kernel in order to be able to formulate Sobolev-Korn estimates on a bounded domain. One the other hand, our aim is to obtain the fundamental solutions for the operator $\operatorname{div} \mathcal{E}$ which can be seen as the generalization of the Laplacian (by definition, div is formally the adjoint to $-\mathcal{E}$ ). These will help to recover a compactly supported tensor field from its symmetrized derivative, a tool that is used to prove compact embedding results.

We first deal with deriving the kernel of the symmetrized derivative on a domain $\Omega \subset \mathbb{R}^{d}$. It will turn out that, for symmetric $k$-tensor fields, it consists of certain polynomials up to the order $k$.

Proposition 3.1 Let $u: \Omega \rightarrow \operatorname{Sym}^{k}\left(\mathbb{R}^{d}\right)$ be $(k+1)$ times continuously differentiable and such that $\mathcal{E}(u)=0$. Then $\nabla^{k+1} \otimes u=0$.

Proof We will prove that

$$
\frac{\partial^{k+1} u\left(e_{i_{1}}, \ldots, e_{i_{k}}\right)}{\partial x_{i_{k+1}} \partial x_{i_{k+2}} \cdots \partial x_{i_{2 k+1}}}=0
$$

for any combination of values/directions $i:\{1, \ldots, 2 k+1\} \rightarrow\{1, \ldots, d\}$. For that purpose, we first introduce an appropriate notation: For each subset $L \subset\{1, \ldots, 2 k+1\}$ with $|L|=k$, we set

$$
u_{L}(x)=\frac{1}{k !} \sum_{\substack{l:\{1, \ldots, k\} \rightarrow L \\ l \text { bijective }}} u(x)\left(e_{i_{l_{1}}}, \ldots, e_{i_{l_{k}}}\right) .
$$

Each summand yields, of course, the same value since $u$ is symmetric meaning that the order of $l_{1}, \ldots, l_{k}$ does not matter, so it suffices to evaluate $u(x)\left(e_{i_{l_{1}}}, \ldots, e_{i_{l_{k}}}\right)$ for one particular bijective $l:\{1, \ldots, k\} \rightarrow L$. Likewise, we define 


$$
\frac{\partial^{k+1} u_{L}}{\partial x_{\complement L}}(x)=\frac{1}{(k+1) !}\left(\sum_{\substack{l:\{1, \ldots, k+1\} \rightarrow \complement L \\ l \text { bijective }}} \frac{\partial^{k+1} u_{L}}{\partial x_{i_{l_{1}}} \cdots \partial x_{i_{l_{k+1}}}}\right)(x)
$$

for which the same as above applies due to the symmetry of the derivative. It should be noted that the expressions $\mathcal{E}(u)_{C L}$ as well as $\partial^{k} / \partial x_{L} \mathcal{E}(u)_{C_{L}}$ can be defined in analogy:

$$
\begin{aligned}
& \mathcal{E}(u)_{\mathrm{C} L}(x)=\frac{1}{(k+1) !} \sum_{\substack{l:\{1, \ldots, k+1\} \rightarrow \complement L \\
l \text { bijective }}} \frac{\partial u}{\partial x_{i_{l_{k+1}}}}(x)\left(e_{i_{l_{1}}}, \ldots, e_{i_{l_{k}}}\right), \\
& \frac{\partial^{k} \mathcal{E}(u)_{\complement} L}{\partial x_{L}}(x)=\frac{1}{(k+1) ! k !} \sum_{\substack{l \in S_{2 k+1} \\
l(\{1, \ldots, k+1\})=\complement}} \frac{\partial^{k+1} u}{\partial x_{i_{l_{k+1}}} \cdots \partial x_{i_{l_{2 k+1}}}}(x)\left(e_{i_{l_{1}}}, \ldots, e_{i_{l_{k}}}\right) . \\
& l(\{k+2, \ldots, 2 k+1\})=L
\end{aligned}
$$

The goal is to obtain the representation

$$
\frac{\partial^{k+1} u_{\{1, \ldots, k\}}}{\partial x_{\complement}\{1, \ldots, k\}}=\sum_{\substack{L \subset\{1, \ldots, 2 k+1\} \\|L|=k}} a_{L} \frac{\partial^{k} \mathcal{E}(u)_{C L}}{\partial x_{L}}
$$

with some coefficients $a_{L} \in \mathbb{R}$ which are independent of the actual choice of $i$. In order to do so, we derive a system of linear equations for $\left\{a_{L}\right\}$ and show that it can be uniquely solved. Let us therefore note that

$$
\begin{aligned}
\frac{\partial^{k} \mathcal{E}(u)_{C L}}{\partial x_{L}} & =\frac{1}{(k+1) ! k !} \sum_{\substack{l \in S_{2 k+1} \\
l(\{1, \ldots, k+1\})=C}} \frac{\partial^{k+1} u}{\partial x_{i_{l_{k+1}}} \cdots \partial x_{i_{l_{2 k+1}}}}\left(e_{i_{l_{1}}}, \ldots, e_{i_{l_{k}}}\right) \\
& =\frac{1}{k+1} \sum_{l_{k+1} \notin L} \frac{1}{(k !)^{2}} \sum_{\substack{l(\{k+2, \ldots, 2 k+1\})=L \\
l(\{1, \ldots, k\})=C L \backslash\left\{l_{k+1}\right\}}} \frac{\partial^{k+1} u}{\partial x_{i_{l_{k+1}}} \cdots \partial x_{i_{l_{2 k+1}}}}\left(e_{i_{l_{1}}}, \ldots, e_{i_{l_{k}}}\right) \\
& =\frac{1}{k+1} \sum_{l_{k+1} \notin L} \frac{1}{(k+1) !} \sum_{\kappa(\{1, \ldots, k+1\})=L \cup\left\{l_{k+1}\right\}} \frac{\partial^{k+1} u_{\complement L} \backslash\left\{l_{k+1}\right\}}{\partial x_{i_{\kappa_{1}}} \cdots \partial x_{\kappa_{k+1}}} \\
& =\frac{1}{k+1} \sum_{l_{k+1} \notin L} \frac{\partial^{k+1} u_{\complement L \backslash\left\{l_{k+1}\right\}}}{\partial x_{L \cup\left\{l_{k+1}\right\}}}=\frac{1}{k+1} \sum_{\substack{M \subset\{1, \ldots, 2 k+1\} \\
|M|=k, L \cap M=\emptyset}} \frac{\partial^{k+1} u_{M}}{\partial x_{\complement} M}
\end{aligned}
$$

so taking $\partial^{k+1} / \partial x_{\mathrm{C} M} u_{M}$ formally as basis vectors (indexed by all subsets $M$ of $\{1, \ldots, 2 k+1\}$ with $k$ elements), we see that (3.1) amounts to

$$
\begin{aligned}
\frac{\partial^{k+1} u_{\{1, \ldots, k\}}}{\partial x_{\complement}\{1, \ldots, k\}} & =\sum_{\substack{L \subset\{1, \ldots, 2 k+1\} \\
|L|=k}} \frac{a_{L}}{k+1} \sum_{\substack{M \subset\{1, \ldots, 2 k+1\} \\
|M|=k, L \cap M=\emptyset}} \frac{\partial^{k+1} u_{M}}{\partial x_{\complement} M} \\
& =\sum_{\substack{M \subset\{1, \ldots, 2 k+1\} \\
|M|=k}}\left(\sum_{\substack{L \subset\{1, \ldots, 2 k+1\} \\
|L|=k, L \cap M=\emptyset}} \frac{a_{L}}{k+1}\right) \frac{\partial^{k+1} u_{M}}{\partial x_{\complement} M} .
\end{aligned}
$$


Now the system is solvable if the matrix given by

$$
E=\left\{E_{L M}\right\}_{L M} \quad, \quad E_{L M}= \begin{cases}\frac{1}{k+1} & L \cap M=\emptyset \\ 0 & \text { else }\end{cases}
$$

is invertible. But $E$ is, up to the factor $1 /(k+1)$, exactly the adjacency matrix associated with the Kneser graph $K_{2 k+1: k}$ (also known as the odd graph $O_{k+1}$ ), see, for instance, [3]. For such adjacency matrices, the eigenvalues are known to be

$$
m=0, \ldots, k: \quad(-1)^{m}\left(\begin{array}{c}
k+1-m \\
k-m
\end{array}\right)=(-1)^{m}(k+1-m) \neq 0,
$$

see [10], and consequently, $E$ is invertible. Hence, there are some $\left\{a_{L}\right\}_{L}$ such that (3.1) is satisfied for each $i:\{1, \ldots, 2 k+1\} \rightarrow\{1, \ldots, d\}$.

Finally, suppose that for some $(k+1)$ times differentiable symmetric $k$-tensor field $u$ we have $\mathcal{E}(u)=0$ in $\Omega$. Then, according to (3.1), we can represent each $(k+1)$ th partial derivative of each component of $u$ (with respect to the canonical basis in $\mathcal{T}^{k}\left(\mathbb{R}^{d}\right)$ ) in terms of a linear combination involving the $k$ th partial derivative of each component of $\mathcal{E}(u)$. As the right-hand side of (3.1) is always zero, the left-hand side also has to vanish what was what we wanted to show.

Proposition 3.2 Let $u: \Omega \rightarrow \operatorname{Sym}^{k}\left(\mathbb{R}^{d}\right)$ be $(k+1)$ times continuously differentiable and such that $\mathcal{E}(u)=0$. Then $u$ is a $\operatorname{Sym}^{k}\left(\mathbb{R}^{d}\right)$-valued polynomial of maximal degree $k$ of the form

$$
u(x)\left(y_{1}, \ldots, y_{k}\right)=\sum_{l=0}^{k} A_{l}(\underbrace{x, \ldots, x}_{l \text { times }}, y_{1}, \ldots, y_{k})
$$

where $A_{l} \in \operatorname{Sym}^{l}\left(\mathbb{R}^{d}\right) \otimes \operatorname{Sym}^{k}\left(\mathbb{R}^{d}\right), l=1, \ldots, k$ such that for each $x_{1}, \ldots, x_{l-1}$, $y_{1}, \ldots, y_{k+1} \in \mathbb{R}^{d}$ it holds that

$$
\sum_{m=1}^{k+1} A_{l}\left(x_{1}, \ldots, x_{l-1}, y_{m}, \ldots, y_{k+1}, y_{1}, \ldots, y_{m-1}\right)=0 \text {. }
$$

Proof From Proposition 3.1, we know that $\nabla^{k+1} \otimes u=0$ from which follows, by Taylor expansion and since $\Omega$ is connected, that $u$ is a $\operatorname{Sym}^{k}\left(\mathbb{R}^{d}\right)$-valued polynomial of maximal degree less than $k+1$. The representation (3.3) is then due to the fact that each $l$-homogeneous polynomial $p_{l}: \Omega \rightarrow \operatorname{Sym}^{k}\left(\mathbb{R}^{d}\right)$ can be represented as

$$
p_{l}(x)\left(y_{1}, \ldots, y_{k}\right)=A_{l}(\underbrace{x, \ldots, x}_{l \text { times }}, y_{1}, \ldots, y_{k})
$$

for some $A_{l} \in \operatorname{Sym}^{l}\left(\mathbb{R}^{d}\right) \otimes \operatorname{Sym}^{k}\left(\mathbb{R}^{d}\right)$. 
It remains to show that (3.4) holds for each $A_{l}$. For that purpose, compute $\mathcal{E}(u)$ for some $x \in \Omega$, taking the symmetry into account:

$$
\begin{aligned}
\mathcal{E}(u)(x)\left(y_{1}, \ldots, y_{k+1}\right) & \\
= & \sum_{l=0}^{k} \frac{1}{k+1} \sum_{m=1}^{k+1} \sum_{i=1}^{d} \frac{\partial}{\partial x_{i}} A_{l}(\underbrace{x, \ldots, x}_{l \text { times }}, y_{m+1}, \ldots, y_{k+1}, y_{1}, \ldots, y_{m-1}) y_{m, i} \\
& =\sum_{l=1}^{k} \frac{l}{k+1} \sum_{m=1}^{k+1} A_{l}(\underbrace{x, \ldots, x}_{(l-1) \text { times }}, y_{m}, \ldots, y_{k+1}, y_{1}, \ldots, y_{m-1})=0 .
\end{aligned}
$$

Since $u$ is a polynomial and $\Omega$ contains an open ball in $\mathbb{R}^{d}$, one can say that each $A_{l}$ with $l=1, \ldots, k$ has to satisfy (3.4) at least for those $x_{1}, \ldots, x_{l-1} \in \mathbb{R}^{d}$ with $x_{1}=\cdots=x_{l-1}$. But, for each $y_{1}, \ldots, y_{k+1}$, the function

$$
\tilde{A}_{l}\left(y_{1}, \ldots, y_{k+1}\right)=\frac{1}{k+1} \sum_{m=1}^{k+1} A_{l}(\underbrace{\cdot, \ldots,}_{(l-1) \text { times }}, y_{m}, \ldots, y_{k+1}, y_{1}, \ldots, y_{m-1})
$$

is a homogeneous polynomial of degree $l-1$ which is identically zero. Since this uniquely determines the underlying element in $\operatorname{Sym}^{l-1}\left(\mathbb{R}^{d}\right)$, we have (3.4) as claimed.

Proposition 3.3 Let $T$ be a distribution on $\Omega$ with $\mathcal{E}(T)=0$ in the distributional sense, i.e. $T: \mathcal{C}_{\mathrm{c}}^{\infty}\left(\Omega, \operatorname{Sym}^{k}\left(\mathbb{R}^{d}\right)\right) \rightarrow \mathbb{R}$ is linear and for each $\Omega^{\prime} \subset \subset \Omega$, there exists a $C_{\Omega^{\prime}}>0$ and an $m_{\Omega^{\prime}} \in \mathbb{N}_{0}$ such that

$$
|T(\varphi)| \leq C_{\Omega^{\prime}}\left\|\nabla^{l} \otimes \varphi\right\|_{\infty} \quad \text { for all } \varphi \in \mathcal{C}_{\mathrm{c}}^{\infty}\left(\Omega^{\prime}, \operatorname{Sym}^{k}\left(\mathbb{R}^{d}\right)\right), 0 \leq l \leq m_{\Omega^{\prime}}
$$

and it holds that

$$
T(\operatorname{div} \varphi)=0 \quad \text { for all } \varphi \in \mathcal{C}_{\mathrm{c}}^{\infty}\left(\Omega, \operatorname{Sym}^{k+1}\left(\mathbb{R}^{d}\right)\right) .
$$

Then, $T$ is a regular distribution in $\mathcal{C}^{\infty}\left(\Omega, \operatorname{Sym}^{k}\left(\mathbb{R}^{d}\right)\right)$ and admits the representation stated in Proposition 3.2.

Proof It suffices to show that there is a $u$ satisfying (3.3) and (3.4) such that for each test function $\varphi \in \mathcal{C}_{\mathrm{c}}^{\infty}\left(\operatorname{Sym}^{k}\left(\mathbb{R}^{d}\right)\right)$, we have

$$
T(\varphi)=\int_{\Omega} u \cdot \varphi \mathrm{d} x .
$$

For this purpose, fix a subdomain $\Omega^{\prime} \subset \subset \Omega$, denote by $G \in \mathcal{C}_{\mathrm{c}}^{\infty}\left(B_{1}(0)\right)$ a standard mollifier and by $G^{\varepsilon}(x)=\varepsilon^{-d} G(x / \varepsilon), \varepsilon>0$, its dilated versions. Observe that there is a $\varepsilon_{0}>0$ such that for each $0<\varepsilon<\varepsilon_{0}$

$$
T^{\varepsilon}(\varphi)=T\left(\varphi * G^{\varepsilon}\right) \quad, \quad \varphi \in \mathcal{C}_{\mathrm{c}}^{\infty}\left(\Omega^{\prime}, \operatorname{Sym}^{k}\left(\mathbb{R}^{d}\right)\right)
$$

defines a distribution on $\Omega^{\prime}$ which is easily verified to satisfy

$$
\left|\nabla^{l} \otimes T^{\varepsilon}(\varphi)\right|=\left|T^{\varepsilon}\left(\operatorname{div}^{l} \varphi\right)\right| \leq C_{\Omega^{\prime}+B_{\varepsilon_{0}}(0)}\left\|\operatorname{div}^{l} G^{\varepsilon}\right\|_{1}\|\varphi\|_{\infty}
$$

for all $\varphi \in \mathcal{C}_{\mathrm{c}}^{\infty}\left(\Omega^{\prime}, \mathcal{T}^{k+l}\left(\mathbb{R}^{d}\right)\right)$ and $l \in \mathbb{N}_{0}$. This means that each $T^{\varepsilon}$ can be represented by $u^{\varepsilon} \in \mathcal{C}^{k+1}\left(\Omega^{\prime}, \operatorname{Sym}^{k}\left(\mathbb{R}^{d}\right)\right)$ in terms of $T^{\varepsilon}(\varphi)=\int_{\Omega} u^{\varepsilon} \cdot \varphi \mathrm{d} x$ for all $\varphi \in \mathcal{C}_{\mathrm{c}}^{\infty}\left(\Omega^{\prime}, \operatorname{Sym}^{k}\left(\mathbb{R}^{d}\right)\right)$. In particular, as $(\operatorname{div} \varphi) * G^{\varepsilon}=\operatorname{div}\left(\varphi * G^{\varepsilon}\right)$, it holds that $\mathcal{E}\left(u^{\varepsilon}\right)=0$ in the weak sense and, 
as $u^{\varepsilon}$ is smooth, also in the strong sense. Employing Proposition 3.2 we deduce that each $u^{\varepsilon}$ obeys (3.3) and (3.4) which also means that all $u^{\varepsilon}$ are contained in a subspace of the finitedimensional space of polynomials of degree less than $k+1$ on $\Omega^{\prime}$. In fact, equipping this space with the norm dual to $L^{\infty}\left(\Omega^{\prime}, \operatorname{Sym}^{k}\left(\mathbb{R}^{d}\right)\right)$, the sequence $\left\{u^{\varepsilon}\right\}$ turns out to be bounded as (3.6) holds independent of $\varepsilon$. By finite dimensionality, $\left\{u^{\varepsilon}\right\}$ is precompact; therefore, there exists a $u: \Omega^{\prime} \rightarrow \operatorname{Sym}^{k}\left(\mathbb{R}^{d}\right)$ satisfying (3.3) and (3.4) such that

$$
T(\varphi)=\lim _{n \rightarrow \infty} T^{\varepsilon_{n}}(\varphi)=\lim _{n \rightarrow \infty} \int_{\Omega^{\prime}} u^{\varepsilon_{n}} \cdot \varphi \mathrm{d} x=\int_{\Omega^{\prime}} u \cdot \varphi \mathrm{d} x
$$

for each $\varphi \in \mathcal{C}_{\mathrm{c}}^{\infty}\left(\Omega^{\prime}, \operatorname{Sym}^{k}\left(\mathbb{R}^{d}\right)\right)$ and at least for a specific sequence $\varepsilon_{n} \rightarrow 0$ as $n \rightarrow \infty$.

Now, such an argumentation can be carried out for each subdomain $\Omega^{\prime} \subset \subset \Omega$ leading to the same $u$ in terms of (3.3): For some subdomains $\Omega_{1}{ }^{\prime}, \Omega_{2}{ }^{\prime} \subset \subset \Omega$ with associated $u_{1}$ and $u_{2}$, it is always possible to choose a subdomain $\Omega^{\prime \prime}$ containing $\Omega_{1}{ }^{\prime} \cup \Omega_{2}{ }^{\prime}$ and, consequently, an associated $u$. By (3.7), $u_{1}=\left.u\right|_{\Omega_{1}{ }^{\prime}}$ and $u_{2}=\left.u\right|_{\Omega_{2}{ }^{\prime}}$ and since $\Omega_{1}{ }^{\prime}$ and $\Omega_{2}{ }^{\prime}$ contain a non-empty open set, respectively, $u, u_{1}$ and $u_{2}$ have to coincide in terms of the representation (3.3). Hence, (3.5) holds of each $\varphi \in \mathcal{C}_{\mathrm{c}}^{\infty}\left(\Omega, \operatorname{Sym}^{k}\left(\mathbb{R}^{d}\right)\right)$ what concludes the proof.

Let us return, for a moment, to the representation of $\nabla^{k+1} \otimes u$ for some smooth $u$ in terms of Eq. (3.1) in the proof of Proposition 3.1. It turns out that the coefficients $\left\{a_{L}\right\}$ can be obtained explicitly.

Proposition 3.4 For $u \in \mathcal{C}^{k+1}\left(\Omega, \operatorname{Sym}^{k}\left(\mathbb{R}^{d}\right)\right)$, we have the representation

$$
\frac{\partial^{k+1} u_{\{1, \ldots, k\}}}{\partial x_{\complement\{1, \ldots, k\}}}=\sum_{\substack{L \subset\{1, \ldots, 2 k+1\} \\
|L|=k}}(-1)^{|L \cap\{1, \ldots, k\}|}\left(\begin{array}{c}
k \\
|L \cap\{1, \ldots, k\}|
\end{array}\right)^{-1} \frac{\partial^{k} \mathcal{E}(u)_{C L}}{\partial x_{L}} .
$$

Proof Recall from the proof of Proposition 3.1 that the above representation is connected with solving a system of linear equations associated with the odd graph $O_{k+1}$. The graphtheoretic distance between some $L, M \subset\{1, \ldots, 2 k+1\}$ with $|L|=|M|=k$ with respect to $O_{k+1}$ can be expressed by

$$
\operatorname{dist}(L, M)=\min \{2|L \cap M|+1,2(k-|L \cap M|)\} .
$$

The odd graph $O_{k+1}$ is known to be distance-regular: For any $L$ and $M$ at distance $m$, the number of vertices adjacent to $M$ at distance $n$ (with respect to $L$ ) is independent from $L$ and $M$. These numbers can be put into the so-called intersection matrix $\left\{B_{m n}\right\}$ for $m, n=0, \ldots, k$ which is tridiagonal by construction. Now, when taking the ansatz that the coefficients $a_{L}$ only depend on $d_{L}=\operatorname{dist}(L,\{1, \ldots, k\})$, then (3.2) becomes

$$
\begin{aligned}
(k+1) \frac{\partial^{k+1} u_{\{1, \ldots, k\}}}{\partial x_{\complement\{1, \ldots, k\}}} & =\sum_{\substack{M \subset\{1, \ldots, 2 k+1\} \\
|M|=k}}\left(\sum_{\substack{L \subset\{1, \ldots, 2 k+1\} \\
|L|=k, L \cap M=\emptyset}} a_{d_{L}}\right) \frac{\partial^{k+1} u_{M}}{\partial x_{\complement} M} \\
& =\sum_{\substack{M \subset\{1, \ldots, 2 k+1\} \\
|M|=k}}\left(\sum_{n=0}^{k} b_{d_{M}, n} a_{n}\right) \frac{\partial^{k+1} u_{M}}{\partial x_{\complement}} \\
& =\sum_{m=0}^{k}\left(\sum_{n=0}^{k} b_{m n} a_{n}\right)\left(\sum_{\substack{M \subset\{1, \ldots, 2 k+1\} \\
|M|=k, d_{M}=m}} \frac{\partial^{k+1} u_{M}}{\partial x_{\complement} M}\right),
\end{aligned}
$$


which means that the equation is true if and only if $a=\left(a_{0}, \ldots, a_{k}\right)$ satisfies $B a=(k+1) e_{0}$ (where $e_{0}$ is standard unit vector associated with the index 0 ). In order to solve this linear equation, we note that for $O_{k+1}$, the intersection matrix is given by (distinguishing odd and even $k$ )

$$
k=2 l: \quad B=\left(\begin{array}{ccccccccc}
0 & 2 l+1 & & & & & & \\
1 & 0 & 2 l & & & & & \\
& 1 & 0 & 2 l & & & & & \\
& & 2 & 0 & 2 l-1 & & & \\
& & & 2 & 0 & 2 l-1 & & \\
& & & & \ddots & \ddots & \ddots & \\
& & & & & & l & 0 & l+1 \\
& & & & & & 0 & l & l+1
\end{array}\right),
$$

see [3].

Hence, $B a=(k+1) e_{0}$ can easily be solved by back substitution. It turns out that

$$
\begin{aligned}
k=2 l: & \left\{\begin{array}{l}
a_{2 n+1}=(-1)^{n}\left(\begin{array}{c}
2 l \\
n
\end{array}\right)^{-1} n=0, \ldots, l-1, \\
a_{2 n}=(-1)^{n}\left(\begin{array}{c}
2 l \\
n
\end{array}\right)^{-1} n=0, \ldots, l,
\end{array}\right. \\
k=2 l+1: & \left\{\begin{array}{l}
a_{2 n+1}=(-1)^{n}\left(\begin{array}{c}
2 l+1 \\
n
\end{array}\right)^{-1} n=0, \ldots, l, \\
a_{2 n}=(-1)^{n+1}\left(\begin{array}{c}
2 l+1 \\
n
\end{array}\right)^{-1} n=0, \ldots, l .
\end{array}\right.
\end{aligned}
$$

Writing the coefficients in terms of $|L \cap M|$ instead of $\operatorname{dist}(L, M)$, i.e.,

$$
|L \cap M|= \begin{cases}k-\operatorname{dist}(L, M) / 2 & \text { if } \operatorname{dist}(L, M) \text { even } \\ (\operatorname{dist}(L, M)-1) / 2 & \text { if } \operatorname{dist}(L, M) \text { odd }\end{cases}
$$

(confer (3.9)) and using the symmetry of the binomial coefficients finally yields (3.8) by plugging in $a_{L}=a_{d_{L}}$ into (3.1).

The subsequent text deals with the derivation of fundamental solutions of $\operatorname{div} \mathcal{E}=-\mathcal{E}^{*} \mathcal{E}$ for symmetric $k$-tensor fields. The following preliminary observation gives a useful expression involving the Laplacian.

Lemma 3.5 Let $\Omega \subset \mathbb{R}^{d}$ be a domain and $u: \Omega \rightarrow \operatorname{Sym}^{k}\left(\mathbb{R}^{d}\right)$ be twice continuously differentiable. Then

$$
\operatorname{div}(\mathcal{E}(u))=\frac{1}{k+1} \Delta u+\frac{k}{k+1} \mathcal{E}(\operatorname{div} u)
$$


Proof Observe that due to the symmetry of $u$ we have

$$
\begin{aligned}
\mathcal{E}(u)\left(a_{1}, \ldots, a_{k+1}\right) & =\|(\nabla \otimes u)\left(a_{1}, \ldots, a_{k+1}\right) \\
& =\frac{1}{k+1} \sum_{l=1}^{k+1}(\nabla \otimes u)\left(a_{l}, a_{1}, \ldots, a_{l-1}, a_{l+1}, \ldots, a_{k+1}\right)
\end{aligned}
$$

so taking the divergence yields

$$
\begin{aligned}
& \operatorname{div}(\mathcal{E}(u))\left(a_{1}, \ldots, a_{k}\right)=\operatorname{tr}(\nabla \otimes \mathcal{E}(u))\left(a_{1}, \ldots, a_{k}\right) \\
& =\frac{1}{k+1}\left(\sum_{i=1}^{d} \frac{\partial^{2} u}{\partial x_{i}^{2}}\left(a_{1}, \ldots, a_{k}\right)+\sum_{i=1}^{d} \sum_{l=1}^{k} \sum_{j=1}^{d} a_{l, j} \frac{\partial^{2} u}{\partial x_{i} \partial x_{j}}\left(a_{1}, \ldots, a_{l-1}, a_{l+1}, \ldots, a_{k}, e_{i}\right)\right) \\
& =\frac{1}{k+1}\left(\Delta u\left(a_{1}, \ldots, a_{k}\right)+\sum_{l=1}^{k} \sum_{j=1}^{d} a_{l, j} \frac{\partial(\operatorname{div} u)}{\partial x_{j}}\left(a_{1}, \ldots, a_{l-1}, a_{l+1}, \ldots, a_{k}\right)\right) \\
& =\frac{1}{k+1}\left(\Delta u\left(a_{1}, \ldots, a_{k}\right)+\sum_{l=1}^{k}(\nabla \otimes(\operatorname{div} u))\left(a_{l}, a_{1}, \ldots, a_{l-1}, a_{l+1}, \ldots, a_{k}\right)\right) \\
& =\frac{1}{k+1} \Delta u\left(a_{1}, \ldots, a_{k}\right)+\frac{k}{k+1} \mathcal{E}(\operatorname{div} u)\left(a_{1}, \ldots, a_{k}\right) .
\end{aligned}
$$

As an immediate consequence, we obtain Korn's inequality for compactly supported symmetric tensor fields and for $p=2$.

Proposition 3.6 Let $u: \mathbb{R}^{d} \rightarrow \operatorname{Sym}^{k}\left(\mathbb{R}^{d}\right)$ be twice continuously differentiable with compact support. Then

$$
\int_{\mathbb{R}^{d}}|\nabla \otimes u|^{2} \mathrm{~d} x \leq(k+1) \int_{\mathbb{R}^{d}}|\mathcal{E}(u)|^{2} \mathrm{~d} x
$$

with equality if and only if $\operatorname{div} u=0$.

Proof Integration by parts (2.3), (3.10) and symmetric integration by parts (2.2) give

$$
\begin{aligned}
\int_{\mathbb{R}^{d}}|\nabla \otimes u|^{2} \mathrm{~d} x & =-\int_{\mathbb{R}^{d}} \Delta u \cdot u \mathrm{~d} x \\
& =-(k+1) \int_{\mathbb{R}^{d}} \operatorname{div}(\mathcal{E}(u)) \cdot u \mathrm{~d} x+k \iint_{\mathbb{R}^{d}} \mathcal{E}(\operatorname{div} u) \cdot u \mathrm{~d} x \\
& =(k+1) \int_{\mathbb{R}^{d}}|\mathcal{E}(u)|^{2} \mathrm{~d} x-k \int_{\mathbb{R}^{d}}|\operatorname{div} u|^{2} \mathrm{~d} x
\end{aligned}
$$

from which the claim follows immediately.

The fundamental solutions can be computed with Fourier methods as it is shown in the following. 
Proposition 3.7 The fundamental solutions of $\operatorname{div}(\mathcal{E}(u))=f$, i.e., $\Gamma_{\eta}^{k}: \mathbb{R}^{d} \rightarrow \operatorname{Sym}^{k}\left(\mathbb{R}^{d}\right)$ such that, in the distributional sense,

$$
\operatorname{div}\left(\mathcal{E}\left(\Gamma_{\eta}^{k}\right)\right)=\delta_{0} \eta
$$

for $\eta \in \operatorname{Sym}^{k}\left(\mathbb{R}^{d}\right)$, are given by

$$
\Gamma_{\eta}^{k}=\sum_{l=0}^{k}(-1)^{l}\left(\begin{array}{l}
k+1 \\
l+1
\end{array}\right) \mathcal{E}^{l}\left(\operatorname{div}^{l}\left(E_{l+1} \eta\right)\right)
$$

where $E_{m}$ denotes the fundamental solution of the $m$-fold iterated Laplacian $\Delta^{m}$, i.e.,

$$
E_{m}(x)= \begin{cases}\frac{(-1)^{d / 2+1}}{2^{2 m-1} \pi^{d / 2}(m-1) !(m-d / 2) !}|x|^{2 m-d} \log |x| & \text { if } 2 m \geq d \\ \frac{(-1)^{m} \Gamma(d / 2-m)}{2^{2 m} \pi^{d / 2}(m-1) !}|x|^{2 m-d} & \text { and d even }\end{cases}
$$

Proof Let us transform the equation $\operatorname{div}(\mathcal{E}(u))=\delta_{0} \eta$ into Fourier space:

$$
\begin{aligned}
& \operatorname{div}(\mathcal{E}(u))\left(a_{1}, \ldots, a_{k}\right)=\frac{1}{k+1}(\Delta u)\left(a_{1}, \ldots, a_{k}\right) \\
&+\frac{1}{k+1} \sum_{i=1}^{d} \frac{\partial}{\partial x_{i}} \sum_{l=1}^{k} \sum_{j=1}^{d} a_{l, j} \frac{\partial u}{\partial x_{j}}\left(a_{1}, \ldots, a_{l-1}, a_{l+1}, \ldots, a_{k}, e_{i}\right) \\
&= \delta_{0} \eta\left(a_{1}, \ldots, a_{k}\right), \\
& \mathcal{F}(\operatorname{div}(\mathcal{E}(u)))(\xi)\left(a_{1}, \ldots, a_{k}\right)=-\frac{|\xi|^{2}}{k+1} \mathcal{F} u(\xi)\left(a_{1}, \ldots, a_{k}\right) \\
& \quad-\frac{1}{k+1} \sum_{i=1}^{d} \xi_{i} \sum_{l=1}^{k}\left(a_{l} \cdot \xi\right) \mathcal{F} u(\xi)\left(a_{1}, \ldots, a_{l-1}, a_{l+1}, \ldots, a_{k}, e_{i}\right) \\
&=\frac{1}{(2 \pi)^{d / 2}} \eta\left(a_{1}, \ldots, a_{k}\right)
\end{aligned}
$$

for almost every $\xi \in \mathbb{R}^{d}$. In order to solve this equation, we introduce a class of linear operators $\mathcal{T}^{k}\left(\mathbb{R}^{d}\right) \rightarrow \mathcal{T}^{k}\left(\mathbb{R}^{d}\right)$. For a subset $L \subset\{1, \ldots, k\}$ with $L=\left\{j_{1}, \ldots, j_{m}\right\}$ and $\xi \in \mathbb{R}^{d}$ define, for $v \in \mathcal{T}^{k}\left(\mathbb{R}^{d}\right)$

$$
\left(\left(\xi \otimes_{L} \xi\right) v\right)\left(a_{1}, \ldots, a_{k}\right)=\left(\prod_{l \in L} a_{l} \cdot \xi\right) \sum_{p: L \rightarrow\{1, \ldots, d\}} \xi_{p} v\left(a_{p}\right)
$$

with

$$
\xi_{p}=\prod_{l \in L} e_{p_{l}} \quad, \quad\left(a_{p}\right)_{i}= \begin{cases}e_{p_{i}} & \text { if } i \in L \\ a_{i} & \text { else }\end{cases}
$$

For $|\xi|=1$ and $L, M \subset\{1, \ldots, k\}$ one can prove that composition respects set union:

$$
\left(\xi \otimes_{L} \xi\right)\left(\xi \otimes_{M} \xi\right)=\left(\xi \otimes_{L \cup M} \xi\right),
$$

see Lemma A.1. In terms of these operators, we reformulate (3.12) to

$$
T_{\xi} \mathcal{F} u(\xi)=\sum_{\substack{L \subset\{1, \ldots, k\} \\|L| \leq 1}}\left(\frac{\xi}{|\xi|} \otimes_{L} \frac{\xi}{|\xi|}\right) \mathcal{F} u(\xi)=-\frac{k+1}{(2 \pi)^{d / 2}|\xi|^{2}} \eta
$$


for almost every $\xi \in \mathbb{R}^{d}$. Surprisingly, these equations have a simple solution with respect to $\mathcal{F} u(\xi)$ : With

$$
S_{\xi} v=\sum_{L \subset\{1, \ldots, k\}} \frac{(-1)^{|L|}}{|L|+1}\left(\frac{\xi}{|\xi|} \otimes_{L} \frac{\xi}{|\xi|}\right)
$$

one sees, with the help of (3.13), that

$$
\begin{aligned}
& S_{\xi} T_{\xi}=\sum_{L \subset\{1, \ldots, k\}} \frac{(-1)^{|L|}}{|L|+1}\left(\frac{\xi}{|\xi|} \otimes_{L} \frac{\xi}{|\xi|}\right) \sum_{\substack{M \subset\{1, \ldots, k\} \\
|M| \leq 1}}\left(\frac{\xi}{|\xi|} \otimes_{M} \frac{\xi}{|\xi|}\right) \\
& =\sum_{\substack{L, M \subset\{1, \ldots, k\} \\
|M| \leq 1}} \frac{(-1)^{|L|}}{|L|+1}\left(\frac{\xi}{|\xi|} \otimes_{L \cup M} \frac{\xi}{|\xi|}\right) \\
& =\sum_{j=0}^{k} \sum_{\substack{L, M \subset\{1, \ldots, k\} \\
|L|=j,|M| \leq 1}} \frac{(-1)^{j}}{j+1}\left(\frac{\xi}{|\xi|} \otimes_{L \cup M} \frac{\xi}{|\xi|}\right) \\
& =\sum_{j=0} \sum_{\substack{L, M \subset\{1, \ldots, k\} \\
|L|=j,|M| \leq 1 \\
L \cup M=L}} \frac{(-1)^{j}}{j+1}\left(\frac{\xi}{|\xi|} \otimes_{L} \frac{\xi}{|\xi|}\right)+\sum_{j=0}^{k-1} \sum_{\substack{L, M \subset\{1, \ldots, k\} \\
L|=j,| M \mid \leq 1 \\
L \cup M \neq L}} \frac{(-1)^{j}}{j+1}\left(\frac{\xi}{|\xi|} \otimes_{L \cup M} \frac{\xi}{|\xi|}\right) .
\end{aligned}
$$

Now, for $L \subset\{1, \ldots, k\},|L|=j$ there are exactly $j+1$ subsets $M \subset\{1, \ldots, k\},|M| \leq 1$ such that $L \cup M=L$. Furthermore, each $L^{\prime} \subset\{1, \ldots, k\},\left|L^{\prime}\right|=j+1 \geq 1$ can be written in exactly $j+1$ ways in the form $L^{\prime}=L \cup M,|L|=j,|M|=1$. Hence,

$$
\begin{aligned}
S_{\xi} T_{\xi} & =\sum_{j=0}^{k} \sum_{\substack{L \subset\{1, \ldots, k\} \\
|L|=j}}(-1)^{j}\left(\frac{\xi}{|\xi|} \otimes_{L} \frac{\xi}{|\xi|}\right)+\sum_{j=1}^{k} \sum_{\substack{L \subset\{1, \ldots, k\} \\
|L|=j}}(-1)^{j+1}\left(\frac{\xi}{|\xi|} \otimes_{L} \frac{\xi}{|\xi|}\right) \\
& =\left(\frac{\xi}{|\xi|} \otimes \emptyset \frac{\xi}{|\xi|}\right)=I .
\end{aligned}
$$

Applying $S_{\xi}$, (3.14) becomes

$$
\begin{aligned}
\mathcal{F} u(\xi) & =-\frac{k+1}{(2 \pi)^{d / 2}|\xi|^{2}} S_{\xi} \eta \\
& =\sum_{L \subset\{1, \ldots, k\}} \frac{(k+1)(-1)^{|L|}}{(|L|+1)}\left(\xi \otimes_{L} \xi\right) \frac{(-1)^{|L|} \eta}{(2 \pi)^{d / 2}\left(-|\xi|^{2}\right)^{|L|+1}}
\end{aligned}
$$

which we are aiming at transforming back to the spatial representation. For this purpose, we use (A.2) from Lemma A.2 as well as

$$
\mathcal{F} E_{m}(\xi)=(2 \pi)^{-d / 2}\left(-|\xi|^{-2}\right)^{m}
$$


for the Fourier transforms for the fundamental solutions of the iterated Laplacians, which have to be interpreted in the sense of distribution pseudo-functions (see [12, p. 288]). Note that in this sense, (A.2) is still valid; hence, one has

$$
u=\sum_{j=0}^{k}(-1)^{j}\left(\begin{array}{c}
k+1 \\
j+1
\end{array}\right) \mathcal{E}^{j}\left(\operatorname{div}^{j}\left(E_{j+1} \eta\right)\right)
$$

which completes the proof.

Corollary 3.8 For $u \in \mathcal{C}_{\mathrm{c}}^{2}\left(\mathbb{R}^{d}, \operatorname{Sym}^{k}\left(\mathbb{R}^{d}\right)\right)$ we have Green's representation formula according to

$$
u(\bar{x}) \cdot \eta=\int_{\mathbb{R}^{d}} \Gamma_{\eta}^{k}(x-\bar{x}) \cdot \operatorname{div}(\mathcal{E}(u))(x) \mathrm{d} x
$$

for all $\bar{x} \in \mathbb{R}^{d}$ and $\eta \in \operatorname{Sym}^{k}\left(\mathbb{R}^{d}\right)$.

Proof Since $u$ is compactly supported and sufficiently smooth and as $T_{\xi}^{*}=T_{\xi}$, the terms in the integral can Fourier transformed leading to

$$
\begin{aligned}
\int_{\mathbb{R}^{d}} \Gamma_{\eta}^{k}(x-\bar{x}) \cdot \operatorname{div}(\mathcal{E}(u))(x) \mathrm{d} x & =\int_{\mathbb{R}^{d}} \mathcal{F}\left(\Gamma_{\eta}^{k}\right)(\xi) \mathrm{e}^{\mathrm{i} \bar{x} \cdot \xi} \cdot \mathcal{F}(\operatorname{div}(\mathcal{E}(u)))(\xi) \mathrm{d} \xi \\
& =\int_{\mathbb{R}^{d}} \mathcal{F}\left(\Gamma_{\eta}^{k}\right)(\xi) \mathrm{e}^{\mathrm{i} \bar{x} \cdot \xi} \cdot T_{\xi} \mathcal{F}(u)(\xi) \mathrm{d} \xi \\
& =\int_{\mathbb{R}^{d}} T_{\xi}^{*} \mathcal{F}\left(\Gamma_{\eta}^{k}\right)(\xi) \cdot \mathcal{F}(u)(\xi) \mathrm{e}^{\mathrm{i} \bar{x} \cdot \xi} \mathrm{d} \xi \\
& =\frac{1}{(2 \pi)^{d / 2}} \int_{\mathbb{R}^{d}} \eta \cdot \mathcal{F}(u)(\xi) \mathrm{e}^{\mathrm{i} \bar{x} \cdot \xi} \mathrm{d} \xi \\
& =u(\bar{x}) \cdot \eta .
\end{aligned}
$$

The section is concluded with the observation that the symmetrized derivative of each fundamental solution is homogeneous. Based on this, Hölder-continuity-like estimates can be established that turn out to be useful for proving compactness properties.

Lemma 3.9 The fundamental solutions $\Gamma_{\eta}^{k}$ are arbitrarily differentiable in $\mathbb{R}^{d} \backslash\{0\}$. Their symmetrized gradient $\mathcal{E}\left(\Gamma_{\eta}^{k}\right)$ is homogeneous of degree $1-d$ and hence locally integrable.

Moreover, for each $s \in] 0,1[$ there is a constant $C>0$ depending on $s, d, k$ and $\eta$ such that for each $x, h \in \mathbb{R}^{d}$ with $x \neq 0$ and $x+h \neq 0$ it holds that

$$
\left|\mathcal{E}\left(\Gamma_{\eta}^{k}\right)(x+h)-\mathcal{E}\left(\Gamma_{\eta}^{k}\right)(x)\right| \leq C|h|^{s}\left(|x+h|^{1-d-s}+|x|^{1-d-s}\right) .
$$

Proof The differentiability of $\Gamma_{\eta}^{k}$ for any order can be deduced directly from (3.11). It is easily verified that the $l$ th derivative of a homogeneous function of degree $m$ is homogeneous of degree $m-l$. Since the operations

$$
\nabla^{l} \otimes \xi \mapsto \operatorname{tr}^{l}\left(\nabla^{l} \otimes \xi\right)=\operatorname{div}^{l}(\xi) \quad, \quad \nabla^{l} \otimes \xi \mapsto \|\left(\nabla^{l} \otimes \xi\right)=\mathcal{E}^{l}(\xi)
$$


are linear, this statement transfers to the $l$ th divergence and symmetrized gradient, respectively. Consequently, if the dimension $d$ is odd or $l<d / 2-1$, then $\mathcal{E}^{l+1}\left(\operatorname{div}^{l}\left(E_{l+1} \eta\right)\right)$ is homogeneous of degree $1-d$.

The case where $d$ is even and $l \geq d / 2-1$ is a bit more complicated because of the logarithmic term: Note that $|x|^{2 l-d+\overline{2}}$ is a homogeneous polynomial of degree $2 l-d+2$ in $x$; hence, $\nabla^{m}|\cdot|^{2 l-d+2}$ is a homogeneous polynomial of degree $2 l-d+2-m$ (where negative degrees indicate the zero polynomial). Likewise, $\nabla^{m}(\log |\cdot|)$ is homogeneous of degree $-m$ for $m \geq 1$. Now, by the product rule and denoting, for subsets $L \subset\{1, \ldots, l\}$, $a_{L}=\left(a_{i_{1}}, \ldots, a_{i_{|L|}}\right)$ where $L=\left\{i_{1}, \ldots, i_{|L|}\right\}$,

$$
\begin{aligned}
\left(\nabla^{l} \otimes\right. & \left.\left(\eta|\cdot|^{2 l-d+2} \log |\cdot|\right)\right)\left(a_{1}, \ldots, a_{k+l}\right) \\
= & \eta\left(a_{l+1}, \ldots, a_{l+k}\right) \sum_{L \subset\{1, \ldots, l\}} \nabla^{l-|L|}|\cdot|^{2 l-d+2}\left(a_{\complement L}\right) \nabla^{|L|}(\log |\cdot|)\left(a_{L}\right) \\
= & \eta\left(a_{l+1}, \ldots, a_{l+k}\right) \nabla^{l}|\cdot|^{2 l-d+2}\left(a_{1}, \ldots, a_{l}\right)(\log |\cdot|) \\
& +\eta\left(a_{l+1}, \ldots, a_{l+k}\right) \sum_{\substack{L \subset\{1, \ldots, L\} \\
L \neq \emptyset}} \nabla^{l-|L|}|\cdot|^{2 l-d+2}\left(a_{\complement L}\right) \nabla^{|L|}(\log |\cdot|)\left(a_{L}\right),
\end{aligned}
$$

hence, we have the representation

$$
\operatorname{div}^{l}\left(E_{l+1} \eta\right)(x)=E_{\eta, \operatorname{div}, 0}^{l}(x) \log |x|+E_{\eta, \operatorname{div}, 1}^{l}(x)
$$

where $E_{\eta, \text { div }, 0}^{l}$ is a homogeneous polynomial of degree $l-d+2$ and $E_{\eta, \text { div, } 1}^{l}$ is homogeneous of degree $l-d+2$. Analogously, one deduces that

$$
\mathcal{E}^{l+1}\left(E_{\eta, \operatorname{div}, 0}^{l} \log |\cdot|\right)(x)=\mathcal{E}^{l+1}\left(E_{\eta, \operatorname{div}, 0}^{l}\right)(x) \log |x|+E_{\eta, \mathcal{E}, \operatorname{div}}^{l}(x)=E_{\eta, \mathcal{E}, \operatorname{div}}^{l}(x)
$$

where $E_{\eta, \mathcal{E} \text {, div }}^{l}$ is a homogeneous function of degree $1-d$ and $\mathcal{E}^{l+1}\left(E_{\eta, \mathrm{div}, 0}^{l}\right)$ vanishes since $E_{\eta, \text { div, } 0}^{l}$ is a homogeneous polynomial of degree $l-d+2$. Together, we get that $\mathcal{E}^{l+1}\left(\operatorname{div}^{l}\left(E_{l+1} \eta\right)\right)$ is homogeneous of degree $1-d$ and consequently the desired statement for the fundamental solution $\Gamma_{\eta}^{k}$.

Now, the estimate (3.16) follows from a general statement on smooth $(1-d)$-homogeneous functions which can, for example, be found in [15, Lemma II.1.4].

\section{Tensor fields of bounded deformation}

Let us first give a precise definition of the space of symmetric tensor fields of bounded deformation. For this, the notion of the "total deformation" of a symmetric $k$-tensor field is first introduced.

Definition 4.1 The total deformation of a $u \in L_{\text {loc }}^{1}\left(\Omega, \operatorname{Sym}^{k}\left(\mathbb{R}^{d}\right)\right)$ is defined as

$$
\mathrm{TD}(\xi)=\sup \left\{\int_{\Omega} \xi \cdot \operatorname{div} \eta \mathrm{d} x \mid \eta \in \mathcal{C}_{\mathrm{c}}^{1}\left(\Omega, \operatorname{Sym}^{k}\left(\mathbb{R}^{d}\right)\right),\|\eta\|_{\infty} \leq 1\right\}
$$


The vector space

$$
\begin{aligned}
\operatorname{BD}\left(\Omega, \operatorname{Sym}^{k}\left(\mathbb{R}^{d}\right)\right) & =\left\{\xi \in L^{1}\left(\Omega, \operatorname{Sym}^{k}\left(\mathbb{R}^{d}\right)\right) \mid \operatorname{TD}(\xi)<\infty\right\}, \\
\|\xi\|_{\mathrm{BD}} & =\|\xi\|_{1}+\mathrm{TD}(\xi)
\end{aligned}
$$

is called the space of symmetric $k$-tensor fields of bounded deformation.

Using the preliminary observations in Sect. 2, it is easy to see that if $\operatorname{TD}(u)<\infty$, then the weak $l$ th symmetrized derivative is a Radon measure on $\Omega$ with values in $\operatorname{Sym}^{k+1}\left(\mathbb{R}^{d}\right)$. It is moreover immediate that $\operatorname{BD}\left(\Omega, \operatorname{Sym}^{k}\left(\mathbb{R}^{d}\right)\right)$ is a normed vector space. Its Banach space property is a consequence of the closedness of $\mathcal{E}$ which we will prove in the following in a weak sense.

Proposition 4.2 The symmetrized gradient is closed in the following sense: Let $p \in[1, \infty]$. If $\xi^{n} \rightarrow \xi$ in $L^{p}\left(\Omega, \operatorname{Sym}^{k}\left(\mathbb{R}^{d}\right)\right)$ as well as $\mathcal{E}\left(\xi^{n}\right) \rightarrow \eta$ in $L^{p}\left(\Omega, \operatorname{Sym}^{k+1}\left(\mathbb{R}^{d}\right)\right)$, then $\mathcal{E}(\xi)=\eta$. The statement remains true for weak ${ }^{*}$-convergence in $\mathcal{M}\left(\Omega, \operatorname{Sym}^{k}\left(\mathbb{R}^{d}\right)\right)$ and $\mathcal{M}\left(\Omega, \operatorname{Sym}^{k+1}\left(\mathbb{R}^{d}\right)\right)$, respectively.

Proof Since each $\zeta \in \mathcal{C}_{\mathrm{c}}^{1}\left(\Omega, \operatorname{Sym}^{k+1}\left(\mathbb{R}^{d}\right)\right)$ is contained in the respective (pre)dual space, we have

$$
\int_{\Omega} \mathcal{E}\left(u^{n}\right) \cdot \zeta \mathrm{d} x \rightarrow \int_{\Omega} \eta \cdot \zeta \mathrm{d} x .
$$

The same can be said for $\operatorname{div} \zeta$ :

$$
\int_{\Omega} \xi^{n} \cdot \operatorname{div} \zeta \mathrm{d} x \rightarrow \int_{\Omega} \xi \cdot \operatorname{div} \zeta \mathrm{d} x .
$$

Consequently, both sides of the defining integral for $\mathcal{E}(u)$ converge, implying that $\mathcal{E}(\xi)=\eta$.

Remark 4.3 We may in particular consider strong convergence instead of weak or weak* convergence.

Proposition 4.4 The space $\operatorname{BD}\left(\Omega, \operatorname{Sym}^{k}\left(\mathbb{R}^{d}\right)\right)$ is a Banach space.

Proof Consider the subspace

$$
\begin{aligned}
U & =\left\{(\xi, \eta) \in L^{1}\left(\Omega, \operatorname{Sym}^{k}\left(\mathbb{R}^{d}\right)\right) \times \mathcal{M}\left(\Omega, \operatorname{Sym}^{k+1}\left(\mathbb{R}^{d}\right)\right) \mid \eta=\mathcal{E}(\xi)\right\} \\
& \subset L^{1}\left(\Omega, \operatorname{Sym}^{k}\left(\mathbb{R}^{d}\right)\right) \times \mathcal{M}\left(\Omega, \operatorname{Sym}^{k+1}\left(\mathbb{R}^{d}\right)\right)
\end{aligned}
$$

for which the mapping $j: \operatorname{BD}\left(\Omega, \operatorname{Sym}^{k}\left(\mathbb{R}^{d}\right)\right) \rightarrow U$ defined by $j(\xi)=(\xi, \mathcal{E}(\xi))$ is an isometrical isomorphism. Now $U$ is closed due to Proposition 4.2 and Remark 4.3; hence, $\operatorname{BD}\left(\Omega, \operatorname{Sym}^{k}\left(\mathbb{R}^{d}\right)\right)$ has to be complete.

The following focusses on estimating the $d /(d-1)$-norm of a symmetric $k$-tensor field in terms of its total deformation according to (4.1). We begin with establishing this estimate for $\mathcal{C}_{\mathrm{c}}^{1}\left(\mathbb{R}^{d}, \operatorname{Sym}^{k}\left(\mathbb{R}^{d}\right)\right)$ which provides the basis for all subsequent estimates.

Lemma 4.5 Let $U \subset S^{d-1}$ be a non-empty, open subset of the unit sphere in $\mathbb{R}^{d}$. Then, there exist $y_{\beta} \in U$ indexed by the multiindices $|\beta|=k$ such that

$$
|\xi|=\left(\sum_{|\beta|=k}\left|\xi\left(y_{\beta}, \ldots, y_{\beta}\right)\right|^{p}\right)^{1 / p}
$$

is equivalent to the Euclidean norm $|\cdot|$ in $\operatorname{Sym}^{k}\left(\mathbb{R}^{d}\right)$ for all $1 \leq p<\infty$. 
In particular, the values of $\xi\left(y_{\beta}, \ldots, y_{\beta}\right)$ for $|\beta|=k$ uniquely determine $\xi \in \operatorname{Sym}^{k}\left(\mathbb{R}^{d}\right)$ and there is a linear mapping $\xi \mapsto\left\{g_{\beta}\right\}$ such that

$$
\xi=\sum_{|\beta|=k} g_{\beta}\left(y_{\beta} \otimes \cdots \otimes y_{\beta}\right)
$$

for all $\xi \in \operatorname{Sym}^{k}\left(\mathbb{R}^{d}\right)$.

Proof Note that, as already pointed out in Sect. 2, there is a one-to-one relation between symmetric $k$-tensors and $k$-homogeneous polynomials on $\mathbb{R}^{d}$. Hence, we shall show that such a polynomial is uniquely determined by the values in the multiindices $\beta \in \mathbb{N}^{d}$ with $|\beta|=k$. Indeed, the following Lagrange basis of polynomials can be constructed: Choose for each $i=1, \ldots, d$ and $j=0, \ldots, k-1$ a vector $h_{i, j} \in S^{d-1}$ for which holds that

$$
h_{i, j} \cdot \beta=0 \quad \text { for all } \beta=j e_{i}+(k-j) e_{l} \text { such that } l \neq i .
$$

Then, with $|\beta|=k$, each function

$$
p_{\beta}(y)=\prod_{i=1}^{d} \prod_{j=0}^{\beta_{i}-1} \frac{y \cdot h_{i, j}}{\beta \cdot h_{i, j}}
$$

gives a $k$-homogeneous polynomial which satisfies $p_{\beta}(\gamma)=\delta_{\beta, \gamma}$ for $|\gamma|=k$ : The identity $p_{\beta}(\beta)=1$ is easily verified while for $\gamma \neq \beta$ note that there has to be an $i$ such that $j=\gamma_{i} \leq \beta_{i}-1$, so with the representation

$$
\gamma=\sum_{l \neq i} \frac{\gamma_{l}}{(k-j)}\left(j e_{i}+(k-j) e_{l}\right)
$$

we get that $\gamma \cdot h_{i, j}=0$ by the choice of $h_{i, j}$ and hence $p_{\beta}(\gamma)=0$. Consequently, there are as many linearly independent $p_{\beta}$ as $\beta$ with $|\beta|=k$, and the $p_{\beta}$ are indeed a basis.

Since $U$ is non-empty, one can choose a $y_{0} \in U$ and a linear mapping $O \in \mathbb{R}^{d \times d}$ which maps $y_{0}$ to $z=(k / d, \ldots, k / d)$ to $y_{0}$ and $\operatorname{span}\left\{y_{0}\right\}^{\perp}$ to $\operatorname{span}\{z\}^{\perp}$ in a way that, for a given $\varepsilon>0,\left|O^{-1} \beta-y_{0}\right|<\varepsilon$ is satisfied for each $|\beta|=k$. Hence, one can achieve, with an appropriate choice of $\varepsilon$, that also $y_{\beta}=O^{-1} \beta /\left|O^{-1} \beta\right|$ lies in $U$ for each $|\beta|=k$. With this, each $k$-homogeneous polynomial $q_{\beta}$ according to $q_{\beta}(y)=\left|O^{-1} \beta\right|^{k} p_{\beta}(O y)$ satisfies

$$
q_{\beta}\left(y_{\gamma}\right)=\left|O^{-1} \beta\right|^{k} p_{\beta}\left(O y_{\gamma}\right)=\frac{\left|O^{-1} \beta\right|^{k}}{\left|O^{-1} \gamma\right|^{k}} p_{\beta}(\gamma)=\delta_{\beta, \gamma}
$$

which implies that the $\left\{q_{\beta}\right\}$ are a Lagrange basis for the space of $k$-homogeneous polynomials with respect to the points $\left\{y_{\beta}\right\}$. Thus, any norm of the respective basis coefficients, which are, for a $\xi \in \operatorname{Sym}^{k}\left(\mathbb{R}^{d}\right)$, given by $\xi\left(y_{\beta}, \ldots, y_{\beta}\right)$, yields an equivalent norm of $\operatorname{Sym}^{k}\left(\mathbb{R}^{d}\right)$, in particular $|\cdot|$ according to (4.2).

To prove the representation (4.3), choose, for each $\beta$, the symmetric $k$-tensor $\eta_{\beta} \in$ $\operatorname{Sym}^{k}\left(\mathbb{R}^{d}\right)$ associated with $q_{\beta}$, i.e., $\eta_{\beta}(y, \ldots, y)=q_{\beta}(y)$ for all $y \in \mathbb{R}^{d}$. These $\left\{\eta_{\beta}\right\}$ are a basis of $\operatorname{Sym}^{k}\left(\mathbb{R}^{d}\right)$; hence, one can set, for $\xi \in \operatorname{Sym}^{k}\left(\mathbb{R}^{d}\right), g_{\beta}=\xi \cdot \eta_{\beta}$ and obtains

$$
\begin{aligned}
\left(\sum_{|\beta|=k} g_{\beta}\left(y_{\beta} \otimes \cdots \otimes y_{\beta}\right)\right) \cdot \eta_{\gamma} & =\sum_{|\beta|=k} g_{\beta} \operatorname{tr}^{k}\left(\left(y_{\beta} \otimes \cdots \otimes y_{\beta}\right) \otimes \eta_{\gamma}\right) \\
& =\sum_{|\beta|=k} g_{\beta} q_{\gamma}\left(y_{\beta}\right)=\xi \cdot \eta_{\gamma}
\end{aligned}
$$


for each $|\gamma|=k$ which implies the desired representation.

Lemma 4.6 For each $d \geq 1$ and $k \geq 1$, there exist vectors $h_{m, n} \in \mathbb{R}^{d}$ where $m=1, \ldots$, $d-1, n=1, \ldots, k+1$ and coefficients $r_{n} \in \mathbb{R}$ such that:

1. For $1 \leq m \leq(d-1)$, the identity

$$
\xi\left(e_{1}, \ldots, e_{1}\right)=\sum_{n=1}^{k+1} r_{n} \xi\left(h_{m, n}, \ldots, h_{m, n}\right)
$$

is satisfied for each $\xi \in \operatorname{Sym}^{k}\left(\mathbb{R}^{d}\right)$.

2. For each $\sigma:\{1, \ldots, d-1\} \rightarrow\{1, \ldots, k+1\}$, the vectors

$$
\left(e_{1}, h_{1, \sigma(1)}, \ldots, h_{d-1, \sigma(d-1)}\right)
$$

are a basis of $\mathbb{R}^{d}$.

Proof We prove that for $h_{m, n}=e_{1}+n e_{m+1}$ with $n=1, \ldots, k+1$, coefficients $r_{n}$ can be found which are independent of $m$ such that (4.4) is satisfied. For this purpose, fix a $1 \leq m \leq(d-1)$ and note that

$$
\xi\left(h_{m, n}, \ldots, h_{m, n}\right)=\sum_{l=0}^{k}\left(\begin{array}{l}
k \\
l
\end{array}\right) n^{l} \xi(\underbrace{e_{1}, \ldots, e_{1}}_{(k-l) \text { times }}, \underbrace{e_{m+1}, \ldots, e_{m+1}}_{l \text { times }}),
$$

for each $n=1, \ldots, k+1$, so (4.4) follows, independent of $m$, if there exist $r_{n}$ such that

$$
\sum_{n=1}^{k+1} r_{n} n^{l}=\delta_{0, l} \quad \text { for } l=0, \ldots, k .
$$

Indeed, this system can be solved uniquely as the associated matrix is the Vandermonde matrix for $\{1,2, \ldots, k+1\}$. Finally, it is easily seen that, given $\sigma:\{1, \ldots, d-1\} \rightarrow$ $\{1, \ldots, k+1\}$, the vectors according to (4.5) form a basis of $\mathbb{R}^{d}$ by looking at its determinant:

$$
\operatorname{det}\left(e_{1}, h_{1, \sigma(1)}, \ldots, h_{d-1, \sigma(d-1)}\right)=\prod_{m=1}^{d-1} \sigma(m) \neq 0 .
$$

Lemma 4.7 Let $d \geq 2, g_{1}, \ldots, g_{d} \in \mathbb{R}^{d}$ be a basis of $\mathbb{R}^{d}$ and $v \in L^{1}\left(\mathbb{R}^{d}\right)$ non-negative. Then, for some $C>0$ which is independent of $v$, it holds that

$$
\int_{\mathbb{R}^{d}}\left(\prod_{i=1}^{d} \int_{\mathbb{R}} v\left(x+s_{i} g_{i}\right) \mathrm{d} s_{i}\right)^{\frac{1}{d-1}} \mathrm{~d} x \leq C\left(\int_{\mathbb{R}^{d}} v(x) \mathrm{d} x\right)^{\frac{d}{d-1}} .
$$

Proof First, observe that we can, by introducing $G y=\sum_{i=1}^{d} y_{i} g_{i}$ and its inverse $G^{-1}$, defining $\tilde{v}(y)=v(G y)$, setting $y=G^{-1} x$ and denoting $\tilde{y}_{i}\left(s_{i}\right)=\left(y_{1}, \ldots, y_{i-1}, s_{i}, y_{i+1}, \ldots, y_{d}\right)$, rewrite

$$
\prod_{i=1}^{d} \int_{\mathbb{R}} v\left(x+s_{i} g_{i}\right) \mathrm{d} s_{i}=\prod_{i=1}^{d} \int_{\mathbb{R}} \tilde{v}\left(\tilde{y}_{i}\left(s_{i}\right)\right) \mathrm{d} s_{i}
$$


We therefore show, in the following,

$$
\int_{\mathbb{R}^{d}}\left(\prod_{i=1}^{d} \int_{\mathbb{R}} \tilde{v}\left(\tilde{y}_{i}\left(s_{i}\right)\right) \mathrm{d} s_{i}\right)^{\frac{1}{d-1}} \mathrm{~d} y \leq\left(\int_{\mathbb{R}^{d}} \tilde{v}(y) \mathrm{d} y\right)^{\frac{d}{d-1}} .
$$

For $d=2$, this immediately follows by interchanging the order of integration. The general case can be deduced by induction, assuming that the above is true for some $d \geq 2$ : Denoting by $\bar{y}=\left(y_{1}, \ldots, y_{d}\right)$ the first $d$ components of a $y \in \mathbb{R}^{d+1}$, we find with the Hölder estimate for the exponents $d$ and $d /(d-1)$ that

$$
\begin{aligned}
& \int_{\mathbb{R}} \int_{\mathbb{R}^{d}}\left(\int_{\mathbb{R}} \tilde{v}\left(\tilde{y}_{d+1}(s)\right) \mathrm{d} s \prod_{i=1}^{d} \int_{\mathbb{R}} \tilde{v}\left(\tilde{y}_{i}\left(s_{i}\right)\right) \mathrm{d} s_{i}\right)^{\frac{1}{d}} \mathrm{~d} \bar{y} \mathrm{~d} y_{d+1} \\
& \leq \int_{\mathbb{R}}\left(\int_{\mathbb{R}^{d}} \int_{\mathbb{R}} \tilde{v}\left(\tilde{y}_{d+1}(s)\right) \mathrm{d} s \mathrm{~d} \bar{y}\right)^{\frac{1}{d}}\left(\int_{\mathbb{R}^{d}}\left(\prod_{i=1}^{d} \int_{\mathbb{R}} \tilde{v}\left(\tilde{y}_{i}\left(s_{i}\right)\right) \mathrm{d} s_{i}\right)^{\frac{1}{d-1}} \mathrm{~d} \bar{y}\right)^{\frac{d-1}{d}} \mathrm{~d} y_{d+1} \\
& \leq\left(\int_{\mathbb{R}^{d+1}} \tilde{v}(y) \mathrm{d} y\right)^{\frac{1}{d}}\left(\int_{\mathbb{R}} \int_{\mathbb{R}^{d}} \tilde{v}\left(\bar{y}, y_{d+1}\right) \mathrm{d} \bar{y} \mathrm{~d} y_{d+1}\right) \\
& =\left(\int_{\mathbb{R}^{d+1}} \tilde{v}(y) \mathrm{d} y\right)^{\frac{d+1}{d}}
\end{aligned}
$$

which proves the inductive step. Finally, denoting by $|\operatorname{det} G|$ the transformation determinant of $G$, we get

$$
\begin{aligned}
& |\operatorname{det} G|^{-1} \int_{\mathbb{R}^{d}}\left(\prod_{i=1}^{d} \int_{\mathbb{R}} v\left(x+s_{i} g_{i}\right) \mathrm{d} s_{i}\right)^{\frac{1}{d-1}} \mathrm{~d} x=\int_{\mathbb{R}^{d}}\left(\prod_{i=1}^{d} \int_{\mathbb{R}} \tilde{v}\left(\tilde{y}_{i}\left(s_{i}\right)\right) \mathrm{d} s_{i}\right)^{\frac{1}{d-1}} \mathrm{~d} y \\
& \leq\left(\int_{\mathbb{R}^{d}} \tilde{v}(y) \mathrm{d} y\right)^{\frac{d}{d-1}}=|\operatorname{det} G|^{-\frac{d}{d-1}}\left(\int_{\mathbb{R}^{d}} v(x) \mathrm{d} x\right)^{\frac{d}{d-1}}
\end{aligned}
$$

from which the assertion immediately follows.

Theorem 4.8 For $k \geq 0$ and $d \geq 1$, there exists a constant $C=C(k, d)$ such that for $u \in \mathcal{C}_{\mathrm{c}}^{1}\left(\mathbb{R}^{d}, \operatorname{Sym}^{k}\left(\mathbb{R}^{d}\right)\right)$ the estimate 


$$
\|u\|_{d /(d-1)} \leq C\|\mathcal{E}(u)\|_{1}
$$

is satisfied.

Proof We will only show the case $d \geq 2$ as the inequality is immediate for $d=1$ and follow the basic idea of Strauss [13,15]. First, consider $u_{1}=u\left(e_{1}, \ldots, e_{1}\right)$. With the help of the $h_{m, n}$ and $r_{n}$ of Lemma 4.6, we can represent $u_{1}$ in $d$ different ways: Denoting $u_{n, m}=$ $u\left(h_{m, n}, \ldots, h_{m, n}\right)$, we have, for almost every $x \in \mathbb{R}^{d}$,

$$
\begin{aligned}
& u_{1}(x)=\int_{-\infty}^{0} \frac{\partial u_{1}}{\partial x_{1}}\left(x+s e_{1}\right) \mathrm{d} s, \\
& u_{1}(x)=\sum_{n=1}^{k+1} r_{n} \int_{-\infty}^{0} \frac{\partial u_{m, n}}{\partial h_{m, n}}\left(x+s h_{m, n}\right) \mathrm{d} s
\end{aligned}
$$

where $m=1, \ldots, d-1$. Now $\frac{\partial u_{1}}{\partial x_{1}}=\mathcal{E}(u)\left(e_{1}, \ldots, e_{1}\right)$ as well as $\frac{\partial u_{m, n}}{\partial h_{m, n}}=\mathcal{E}(u)\left(h_{m, n}, \ldots\right.$, $\left.h_{m, n}\right)$, so it follows, again almost everywhere,

$$
\begin{aligned}
& \left|u_{1}(x)\right| \leq \int_{\mathbb{R}}\left|\mathcal{E}(u)\left(x+s e_{1}\right)\right| \mathrm{d} s, \\
& \left|u_{1}(x)\right| \leq C \sum_{n=1}^{k+1} \int_{\mathbb{R}}\left|\mathcal{E}(u)\left(x+s h_{m, n}\right)\right| \mathrm{d} s
\end{aligned}
$$

and consequently,

$$
\begin{aligned}
&\left|u_{1}(x)\right|^{d} \leq C \int_{\mathbb{R}}\left|\mathcal{E}(u)\left(x+s e_{1}\right)\right| \mathrm{d} s \prod_{m=1}^{d-1} \sum_{n=1}^{k+1} \int_{\mathbb{R}}\left|\mathcal{E}(u)\left(x+s h_{m, n}\right)\right| \mathrm{d} s \\
&=C \sum_{\sigma \in\{1, \ldots, k+1\}^{d-1}} \int_{\mathbb{R}}\left|\mathcal{E}(u)\left(x+s_{1} e_{1}\right)\right| \mathrm{d} s_{1} \\
& \times \prod_{i=1}^{d-1} \int_{\mathbb{R}}\left|\mathcal{E}(u)\left(x+s_{i+1} h_{i, \sigma(i)}\right)\right| \mathrm{d} s_{i+1} .
\end{aligned}
$$

Applying the $(d-1)$ th root, estimating the root of the sum by the sum of the roots, integrating and denoting by $g_{\sigma, i}$ with $i=1, \ldots, d$ the basis according to (4.5), it follows that

$$
\int_{\mathbb{R}^{d}}\left|u_{1}(x)\right|^{\frac{d}{d-1}} \mathrm{~d} x \leq C \sum_{\sigma \in\{1, \ldots, k+1\}^{d-1}} \int_{\mathbb{R}^{d}}\left(\prod_{i=1}^{d} \int_{\mathbb{R}}\left|\mathcal{E}(u)\left(x+s_{i} g_{\sigma, i}\right)\right| \mathrm{d} s_{i}\right)^{\frac{1}{d-1}} \mathrm{~d} x
$$

so one can apply Lemma 4.7 to get

$$
\int_{\mathbb{R}^{d}}\left|u_{1}(x)\right|^{\frac{d}{d-1}} \mathrm{~d} x \leq C\left(\int_{\mathbb{R}^{d}}|\mathcal{E}(u)(x)| \mathrm{d} x\right)^{\frac{d}{d-1}}
$$

and hence $\left\|u_{1}\right\|_{d /(d-1)} \leq C\|\mathcal{E}(u)\|_{1}$. 
Now choose $\left\{y_{\beta}\right\}$ according to Lemma 4.5 (with $U=S^{d-1}$, for instance) and note that for each of these $y_{\beta}$, there is an orthonormal matrix $O_{\beta}$ such that $O_{\beta} e_{1}=y_{\beta}$. Furthermore, $\left|\mathcal{E}\left(u \circ O_{\beta}\right)(x) O_{\beta}\right|=\left|\mathcal{E}(u)\left(O_{\beta} x\right)\right|$ (see Lemmas A.4 and A.3), so change of variables $x \mapsto$ $O_{\beta} x$ gives, with the norm equivalence result in Lemma 4.5,

$$
\begin{aligned}
\|u\|_{d /(d-1)} & \leq C\left(\int_{\mathbb{R}^{d}}\left(\sum_{|\beta|=k}\left|u(x)\left(y_{\beta}, \ldots, y_{\beta}\right)\right|\right)^{\frac{d}{d-1}} \mathrm{~d} x\right)^{\frac{d-1}{d}} \\
& \leq C \sum_{|\beta|=k}\left(\int_{\mathbb{R}^{d}}\left|\left(u\left(O_{\beta} x\right) O_{\beta}\right)\left(e_{1}, \ldots, e_{1}\right)\right|^{\frac{d}{d-1}} \mathrm{~d} x\right)^{\frac{d-1}{d}} \\
& \leq C \sum_{|\beta|=k_{\mathbb{R}^{d}}}\left|\mathcal{E}(u)\left(O_{\beta} x\right)\right| \mathrm{d} x \leq C\|\mathcal{E}(u)\|_{1}
\end{aligned}
$$

which concludes the proof.

In the following, we address some density properties for $\operatorname{BD}\left(\Omega, \operatorname{Sym}^{k}\left(\mathbb{R}^{d}\right)\right)$ which allow us to extend the obtained inequalities to symmetric tensor fields of bounded deformation.

Lemma 4.9 $A u \in L^{1}\left(\Omega, \operatorname{Sym}^{k}\left(\mathbb{R}^{d}\right)\right)$ is of bounded deformation if and only if for each $y \in S^{d-1}$ and $u_{y}(x)=u(x)(y, \ldots, y)$, the function satisfies $\frac{\partial u_{y}}{\partial y} \in \mathcal{M}(\Omega)$. For each $y \in S^{d-1}$ we have $\left|\frac{\partial u_{y}}{\partial y}\right| \leq|\mathcal{E}(u)|$ in the sense of inequality of Radon measures.

Moreover, each $\frac{\partial u_{y}}{\partial y}$ can be disintegrated such that for each Borel set $A \subset \Omega$, it holds that

$$
\frac{\partial u_{y}}{\partial y}(A)=\int_{\{y\}^{\perp}} u_{y}(x)^{\prime}\left(A_{x}\right) \mathrm{d} \mathcal{H}^{d-1}(x)
$$

where $u_{y}(x)^{\prime} \in \mathcal{M}(\mathbb{R})$ for $\mathcal{H}^{d-1}$-almost every $x \in \operatorname{span}\{y\}^{\perp}$ and $A_{x}=\{t \in \mathbb{R} \mid x+$ ty $\in A\}$.

Proof For $y \in S^{d-1}$, we can construct the symmetric tensor $\xi_{y}=y \otimes \cdots \otimes y \in \operatorname{Sym}^{k+1}\left(\mathbb{R}^{d}\right)$ which obeys $\left|\xi_{y}\right|=1$. Now, if $v \in \mathcal{C}_{\mathrm{c}}^{1}(\Omega)$ and $\|v\|_{\infty} \leq 1$, then

$$
\int_{\Omega} u_{y} \frac{\partial v}{\partial y} \mathrm{~d} x=\int_{\Omega} u \cdot \operatorname{div}\left(\xi_{y} v\right) \mathrm{d} x
$$

which implies that $\frac{\partial u_{y}}{\partial y} \in \mathcal{M}(\Omega)$ with the inequality $\left|\frac{\partial u_{y}}{\partial y}\right| \leq|\mathcal{E}(u)|$ whenever $u \in$ $\operatorname{BD}\left(\Omega, \operatorname{Sym}^{k}\left(\mathbb{R}^{d}\right)\right)$. For the opposite direction, we write, with the help of Lemma 4.5 , a continuously differentiable $v: \Omega \rightarrow \operatorname{Sym}^{k+1}\left(\mathbb{R}^{d}\right)$ with compact support according to

$$
v(x)=\sum_{|\beta|=k+1} g_{\beta}(x)(\underbrace{y_{\beta} \otimes \cdots \otimes y_{\beta}}_{(k+1) \text { times }}), \quad(\operatorname{div} v)(x)=\sum_{|\beta|=k+1} \frac{\partial g_{\beta}}{\partial y_{\beta}}(x)(\underbrace{y_{\beta} \otimes \cdots \otimes y_{\beta}}_{k \text { times }}),
$$

which implies that 


$$
\begin{aligned}
\int_{\Omega} u \cdot \operatorname{div} v \mathrm{~d} x & =\sum_{|\beta|=k+1} \int_{\Omega} u_{y_{\beta}}(x) \frac{\partial g_{\beta}}{\partial y_{\beta}}(x) \mathrm{d} x \leq \sum_{|\beta|=k+1}\left\|\frac{\partial u_{y_{\beta}}}{\partial y_{\beta}}\right\|_{\mathcal{M}}\left\|g_{\beta}\right\|_{\infty} \\
& \leq C \sum_{|\beta|=k+1}\left\|\frac{\partial u_{y_{\beta}}}{\partial y_{\beta}}\right\|_{\mathcal{M}}<\infty
\end{aligned}
$$

since $\left\|g_{\beta}\right\|_{\infty} \leq\left|\eta_{\beta}\right|\|v\|_{\infty} \leq C\|v\|_{\infty}$ with the $\eta_{\beta}$ chosen in Lemma 4.5. Hence, $\mathcal{E}(u) \in$ $\mathcal{M}\left(\Omega, \operatorname{Sym}^{k+1}\left(\mathbb{R}^{d}\right)\right)$.

Regarding the remainder, from $u_{y} \in L^{1}(\Omega)$ and $\frac{\partial u_{y}}{\partial y} \in \mathcal{M}(\Omega)$ follows that $t \mapsto u_{y}(x+t y)$ is of bounded variation (on the set $\{t \in \mathbb{R} \mid x+t y \in \Omega\}$ ) for almost every $x \in \operatorname{span}\{y\}^{\perp}$, see [1, Theorem 3.103]. Hence, the derivative $u_{y}(x)^{\prime}$ can be extended to a Radon measure in $\mathcal{M}(\mathbb{R})$ for $\mathcal{H}^{d-1}$-almost every $x \in\{y\}^{\perp}$. For $v: \Omega \rightarrow \mathbb{R}$ differentiable with compact support, we then have

$$
\begin{aligned}
\int_{\Omega} v \mathrm{~d} \frac{\partial u_{y}}{\partial y}(x) & =-\int_{\Omega} u_{y} \frac{\partial v}{\partial y} \mathrm{~d} x \\
& =-\int_{\{y\}^{\perp}} \int_{\mathbb{R}} u_{y}(x+t y) \frac{\partial v}{\partial y}(x+t y) \mathrm{d} t \mathrm{~d} \mathcal{H}^{d-1}(x) \\
& =\int_{\{y\}^{\perp}} \int_{\mathbb{R}} v(x+t y) \mathrm{d} u_{y}(x)^{\prime}(t) \mathrm{d} \mathcal{H}^{d-1}(x)
\end{aligned}
$$

which implies the desired representation formula.

Corollary 4.10 One can see analogously that for a sequence $\left\{u^{n}\right\}$ in the space $\mathrm{BD}\left(\Omega, \operatorname{Sym}^{k}\left(\mathbb{R}^{d}\right)\right)$ and some $u \in \mathrm{BD}\left(\Omega, \operatorname{Sym}^{k}\left(\mathbb{R}^{d}\right)\right)$ the convergence $\mathcal{E}\left(u^{n}\right) \stackrel{*}{\rightarrow} \mathcal{E}(u)$ is equivalent to $\frac{\partial u_{y}^{n}}{\partial y} \stackrel{*}{\rightarrow} \frac{\partial u_{y}}{\partial y}$ in $\mathcal{M}(\Omega)$ for each $y \in S^{d-1}$.

Lemma 4.11 Let $y_{0} \in S^{d-1}, F: \operatorname{span}\left\{y_{0}\right\}^{\perp} \rightarrow \operatorname{span}\left\{y_{0}\right\}$ a Lipschitz continuous mapping and $V \subset \operatorname{span}\left\{y_{0}\right\}^{\perp}$ a domain. Denote by $\Gamma=\{\bar{y}+F(\bar{y}) \mid \bar{y} \in V\}$ and let $y \in S^{d-1}$ be such that $\left|y-y_{0}\right|<\left(2\left(\|\nabla F\|_{\infty}+1\right)\right)^{-1}$ with $\|\nabla F\|_{\infty}$ being the Lipschitz constant of $F$. Furthermore, let $\Omega=\Gamma+]-t_{0}, 0\left[y\right.$ and $v \in \mathcal{M}\left(\mathbb{R}^{d}\right)$ such that for each Borel set $A \subset \mathbb{R}^{d}$ it holds that

$$
v(A)=\int_{\{y\}^{\perp}} \bar{v}(x)\left(A_{x}\right) \mathrm{d} \mathcal{H}^{d-1}(x)
$$

with $\bar{v}(x) \in \mathcal{M}(\mathbb{R})$ for $\mathcal{H}^{d-1}$-almost every $x \in \operatorname{span}\{y\}^{\perp}$ and $A_{x}=\{t \mid x+$ ty $\in A\}$. Then, there exists a constant $C>0$ depending only on $\|\nabla F\|_{\infty}$ such that

$$
\int_{\Gamma}\left|\bar{v}\left(P_{y}^{\perp} x\right)\right|(] x \cdot y-t_{0}, x \cdot y[) \mathrm{d} \mathcal{H}^{d-1}(x) \leq C|v|(\Omega)
$$

where $P_{y}^{\perp}$ is the orthogonal projection on the orthogonal complement of $\operatorname{span}\{y\}$.

Proof First note that $Q(\bar{y})=P_{y}^{\perp}(\bar{y}+F(\bar{y}))$ defines a Lipschitz continuous mapping $G$ : $V \rightarrow \operatorname{span}\{y\}^{\perp}$. It is moreover invertible with Lipschitz continuous inverse: For $\bar{y}_{1}, \bar{y}_{2} \in V$, it follows, as $\left|y_{0}\right| \leq 1,|y| \leq 1$ and $V \subset \operatorname{span}\left\{y_{0}\right\}^{\perp}$, that 


$$
\begin{aligned}
\left|Q\left(\bar{y}_{1}\right)-Q\left(\bar{y}_{2}\right)\right|^{2}= & \left|\bar{y}_{1}-\bar{y}_{2}\right|^{2}+\left|F\left(\bar{y}_{1}\right)-F\left(\bar{y}_{2}\right)\right|^{2} \\
& -\left(\left(\bar{y}_{1}-\bar{y}_{2}\right) \cdot\left(y-y_{0}\right)+\left(F\left(\bar{y}_{1}\right)-F\left(\bar{y}_{2}\right)\right) \cdot y\right)^{2} \\
\geq & \left|\bar{y}_{1}-\bar{y}_{2}\right|^{2}-\left|\bar{y}_{1}-\bar{y}_{2}\right|^{2}\left|y-y_{0}\right|^{2} \\
& -2\|\nabla F\|_{\infty}\left|\bar{y}_{1}-\bar{y}_{2}\right|^{2}\left|y-y_{0}\right| \geq c\left|\bar{y}_{1}-\bar{y}_{2}\right|^{2}
\end{aligned}
$$

for some $c>0$ since we have

$$
\left|y-y_{0}\right|^{2}+2\|\nabla F\|_{\infty}\left|y-y_{0}\right| \leq 2\left(\|\nabla F\|_{\infty}+1\right)\left|y-y_{0}\right|=1-c<1
$$

according to the assumption. Therefore, $\tilde{V}=Q(V)$ is a domain in $\operatorname{span}\{y\}^{\perp}$ and $G: \tilde{V} \rightarrow$ $\operatorname{span}\{y\}$ according to

$$
G(\tilde{y})=y \cdot\left(Q^{-1}(\tilde{y})+F\left(Q^{-1}(\tilde{y})\right)\right) y
$$

is also a Lipschitz mapping satisfying $\Gamma=\{\tilde{y}+G(\tilde{y}) \mid \tilde{y} \in \tilde{V}\}$.

Now, the set-function

$$
A \mapsto \int_{\Gamma} \bar{v}\left(P_{y}^{\perp} x\right)\left(\tilde{A}_{x}\right) \mathrm{d} \mathcal{H}^{d-1}(x), \quad \tilde{A}_{x}=\{t \mid(x, t-x \cdot y) \in A\}
$$

defines a Radon measure on $\Gamma \times]-t_{0}, 0[$, we therefore conclude by the area formula, the fact that continuous functions are preserved under Lipeomorphims as well as setting $C=\left(1+\|\nabla G\|_{\infty}^{2}\right)^{1 / 2}$ that

$$
\begin{aligned}
\int_{\Gamma}\left|\bar{v}\left(P_{y}^{\perp} x\right)\right|(] x \cdot y-t_{0}, x \cdot y[) \mathrm{d} \mathcal{H}^{d-1}(x) \\
=\sup _{\substack{\varphi \in \mathcal{C}_{\mathrm{c}}(\Gamma \times]-t_{0}, 0[) \\
\|\varphi\|_{\infty} \leq 1}} \int_{\Gamma} \int_{\mathbb{R}} \varphi(x, t-x \cdot y) \mathrm{d} \bar{v}\left(P_{y}^{\perp} x\right)(t) \mathrm{d} \mathcal{H}^{d-1}(x) \\
=\sup _{\substack{\varphi \in \mathcal{C}_{\mathrm{C}}(\tilde{V} \times]-t_{0}, 0[) \\
\|\varphi\|_{\infty} \leq 1}} \int_{\tilde{V}} \varphi(\tilde{y}, t-G(\tilde{y}) \cdot y) \mathrm{d} \bar{v}(\tilde{y}) \sqrt{1+|\nabla G(\tilde{y})|^{2}} \mathrm{~d} \mathcal{H}^{d-1}(\tilde{y}) \\
\quad \leq \sup _{\substack{\varphi \in \mathcal{C}_{\mathrm{c}}(\Omega) \\
\|\varphi\|_{\infty} \leq C}} \int_{\{y\}^{\perp}} \int_{\mathbb{R}} \varphi(\tilde{y}+t y) \mathrm{d} \bar{v}(\tilde{y}) \mathrm{d} \mathcal{H}^{d-1}(\tilde{y})=C|v|(\Omega) .
\end{aligned}
$$

Theorem 4.12 Let $\Omega$ be a bounded Lipschitz domain. There exists a constant $C>0$ such that the trace mapping $\gamma: \operatorname{BD}\left(\Omega, \operatorname{Sym}^{k}\left(\mathbb{R}^{d}\right)\right) \rightarrow L^{1}\left(\partial \Omega, \operatorname{Sym}^{k}\left(\mathbb{R}^{d}\right)\right)$ is well defined and satisfies the estimate

$$
\|\gamma u\|_{1, \partial \Omega} \leq C\left(\|u\|_{1}+\|\mathcal{E}(u)\|_{\mathcal{M}}\right)
$$

for each $u \in \operatorname{BD}\left(\Omega, \operatorname{Sym}^{k}\left(\mathbb{R}^{d}\right)\right)$.

Proof Since $\Omega$ is a Lipschitz domain, it suffices to restrict ourselves to the following setting. Let $y_{0} \in S^{d-1}$ and $F:\left\{y_{0}\right\}^{\perp} \rightarrow \operatorname{span}\left\{y_{0}\right\}$ a Lipschitz function such that for some domain $V \subset\left\{y_{0}\right\}^{\perp}$ and some $t_{0}>0$, we have 


$$
\begin{gathered}
\bar{y} \in V: \quad \bar{y}+F(\bar{y}) \in \partial \Omega, \\
\bar{y} \in V, t \in] 0, t_{0}\left[: \quad \bar{y}+F(\bar{y})-t y_{0} \in \Omega,\right. \\
\bar{y} \in V, t \in] 0, t_{0}\left[: \quad \bar{y}+F(\bar{y})+t y_{0} \in \mathbb{R}^{d} \backslash \Omega .\right.
\end{gathered}
$$

As Lipschitz domains further satisfy the cone property, we can assume that there is a $\delta>0$ such that for each $y \in S^{d-1}$ with $\left|y-y_{0}\right|<\delta$, the set $\{\bar{y}+F(\bar{y})-t y \mid \bar{y} \in V, t \in] 0, t_{0}[\}$ is contained in $\Omega$.

In the following, let $\Gamma=\{\bar{y}+F(\bar{y}) \mid \bar{y} \in V\}$ on which we will define the trace. Letting $U=\left\{y \in S^{d-1}|| y-y_{0} \mid<\min \left(\delta, \frac{1}{2}\left(1+\|\nabla F\|_{\infty}\right)^{-1}\right)\right\}$, which is a non-empty subset of $S^{d-1}$, we choose $\left\{y_{\beta}\right\}$ in $U$ indexed by the multiindices $\beta$ such that $|\beta|=k$ according to Lemma 4.5. Denote by $\left.\Omega_{\beta}=\Gamma-\right] 0, t_{0}\left[y_{\beta} \subset \Omega\right.$ and by $u_{\beta}(x)=u(x)\left(y_{\beta}, \ldots, y_{\beta}\right)$ for $x \in \Omega_{\beta}$. According to Lemma 4.9, $u_{\beta} \in L^{1}\left(\Omega_{\beta}\right)$ with $\frac{\partial u_{\beta}}{\partial y_{\beta}} \in \mathcal{M}\left(\Omega_{\beta}\right)$, with $t \mapsto u_{\beta}\left(x-t y_{\beta}\right)$ being of bounded variation on $] 0, t_{0}\left[\right.$ for $\mathcal{H}^{d-1}$-almost every $x \in \Gamma$. Hence, one can define a trace $\gamma u_{\beta}(x)$ for almost every $x \in \Gamma$ with

$$
\left|\gamma u_{\beta}(x)\right| \leq \int_{0}^{t_{0}}\left|u_{\beta}\left(x-t y_{\beta}\right)\right| \mathrm{d} t+\left|u_{\beta}\left(P_{y_{\beta}}^{\perp} x\right)^{\prime}\right|(] x \cdot y_{\beta}-t_{0}, x \cdot y_{\beta}[)
$$

where $u_{\beta}(x)^{\prime}$ denotes the derivative of $t \mapsto u_{\beta}\left(x-t y_{\beta}\right)$ extended to $\mathcal{M}(\mathbb{R})$. In case $u_{\beta} \in$ $\mathcal{C}\left(\bar{\Omega}, \operatorname{Sym}^{k}\left(\mathbb{R}^{d}\right)\right)$, we have, of course, $\gamma u_{\beta}(x)=u_{\beta}(x)$ so $\gamma u_{\beta}(x)$ is indeed a trace which will, in the following, just be denoted by $u_{\beta}(x)$.

Consequently, according to the Lemmas 4.9 and 4.11 ,

$$
\begin{aligned}
\int_{\Gamma}\left|\gamma u_{\beta}(x)\right| \mathrm{d} \mathcal{H}^{d-1}(x) & \leq C\left(\int_{\Omega_{\beta}}\left|u_{\beta}\right| \mathrm{d} x+\left|\frac{\partial u_{\beta}}{y_{\beta}}\right|\left(\Omega_{\beta}\right)\right) \\
& \leq C\left(\int_{\Omega_{\beta}}|u(x)| \mathrm{d} x+|\mathcal{E}(u)|\left(\Omega_{\beta}\right)\right)
\end{aligned}
$$

where $C$ can be chosen independent of $\beta$. The collection $\left\{\gamma u_{\beta}\right\}$ for $|\beta|=k$ now defines a $u \in L^{1}\left(\Gamma, \operatorname{Sym}^{k}\left(\mathbb{R}^{d}\right)\right)$, see again Lemma 4.5. With the above, we are able to estimate

$$
\int_{\Gamma}|\gamma u(x)| \mathrm{d} \mathcal{H}^{d-1}(x) \leq C \sum_{|\beta|=k} \int_{\Gamma}\left|\gamma u_{\beta}(x)\right| \mathrm{d} \mathcal{H}^{d-1}(x) \leq C\left(\|u\|_{1}+\|\mathcal{E}(u)\|_{\mathcal{M}}\right) .
$$

Proceeding analogously for each element for the finite covering of the boundary, one arrives at the desired global result.

Proposition 4.13 Let $\Omega$ be a bounded domain. The set $\mathcal{C}^{\infty}\left(\Omega, \operatorname{Sym}^{k}\left(\mathbb{R}^{d}\right)\right) \cap$ $\operatorname{BD}\left(\Omega, \operatorname{Sym}^{k}\left(\mathbb{R}^{d}\right)\right)$ is dense in $\operatorname{BD}\left(\Omega, \operatorname{Sym}^{k}\left(\mathbb{R}^{d}\right)\right)$ in the following sense:

For each $u \in \operatorname{BD}\left(\Omega, \operatorname{Sym}^{k}\left(\mathbb{R}^{d}\right)\right)$ there exists an approximating sequence $\left\{u^{n}\right\}$ in $\mathcal{C}^{\infty}\left(\Omega, \operatorname{Sym}^{k}\left(\mathbb{R}^{d}\right)\right)$ that converges strictly to $u$, i.e., 


$$
\begin{cases}u^{n} \rightarrow u & \text { in } L^{1}\left(\Omega, \operatorname{Sym}^{k}\left(\mathbb{R}^{d}\right)\right), \\ \mathcal{E}\left(u^{n}\right) \stackrel{*}{\rightarrow} \mathcal{E}(u) & \text { in } \mathcal{M}\left(\Omega, \operatorname{Sym}^{k+1}\left(\mathbb{R}^{d}\right)\right), \\ \left\|\mathcal{E}\left(u^{n}\right)\right\|_{\mathcal{M}} \rightarrow\|\mathcal{E}(u)\|_{\mathcal{M}} . & \end{cases}
$$

If the support of $u$ is compact in $\Omega$, then $\left\{u^{n}\right\}$ can be chosen such that each $u^{n}$ is in $\mathcal{C}_{\mathrm{c}}^{\infty}\left(\Omega, \operatorname{Sym}^{k}\left(\mathbb{R}^{d}\right)\right)$.

Proof Note that we can proceed analogously to Theorem 2 in Sect. 5.2.2 in [8] and use mollifiers to obtain a sequence $\left\{u^{n}\right\}$ in $\mathcal{C}^{\infty}\left(\Omega, \operatorname{Sym}^{k}\left(\mathbb{R}^{d}\right)\right)$ with $u^{n} \rightarrow u$ in $L^{1}\left(\Omega, \operatorname{Sym}^{k}\left(\mathbb{R}^{d}\right)\right)$ as well as $\left\|\mathcal{E}\left(u^{n}\right)\right\|_{\mathcal{M}} \rightarrow\|\mathcal{E}(u)\|_{\mathcal{M}}$. In particular, if $u$ has compact support in $\Omega$, then $u^{n} \in \mathcal{C}_{\mathrm{c}}^{\infty}\left(\Omega, \operatorname{Sym}^{k}\left(\mathbb{R}^{d}\right)\right)$ for each $n$.

Now, the convergence properties for $\left\{u^{n}\right\}$ immediately imply that $\mathcal{E}\left(u^{n}\right) \stackrel{*}{\rightarrow} \mathcal{E}(u)$ at least for a subsequence: Since $\left\{\mathcal{E}\left(u^{n}\right)\right\}$ is bounded in $\mathcal{M}\left(\Omega, \operatorname{Sym}^{k+1}\left(\mathbb{R}^{d}\right)\right)$, there exists a weak*-convergent subsequence (still denoted by $\left\{\mathcal{E}\left(u^{n}\right)\right\}$ ) whose limit has to be $\mathcal{E}(u)$ by the closedness of the operator $\mathcal{E}$.

Theorem 4.14 Let $\Omega$ be a bounded Lipschitz domain. Then, the trace operator $\gamma$ : $\mathrm{BD}\left(\Omega, \operatorname{Sym}^{k}\left(\mathbb{R}^{d}\right)\right) \rightarrow L^{1}\left(\partial \Omega, \operatorname{Sym}^{k}\left(\mathbb{R}^{d}\right)\right)$ is continuous with respect to strict convergence and the divergence theorem

$$
\int_{\Omega} u \cdot \operatorname{div} v \mathrm{~d} x=\int_{\partial \Omega} \|(\gamma u \otimes v) \cdot v \mathrm{~d} \mathcal{H}^{d-1}(x)-\int_{\Omega} \mathcal{E}(u) \cdot v \mathrm{~d} x
$$

holds for $u \in \operatorname{BD}\left(\Omega, \operatorname{Sym}^{k}\left(\mathbb{R}^{d}\right)\right)$ and $v \in \mathcal{C}^{1}\left(\bar{\Omega}, \operatorname{Sym}^{k+1}\left(\mathbb{R}^{d}\right)\right)$.

Proof Suppose that the sequence $\left\{u^{n}\right\}$ converges to $u$ in the sense of (4.8). We first like to show that the traces satisfy $\gamma u^{n} \rightarrow \gamma u$ in $L^{1}(\partial \Omega)$ following the lines of proof in [1, Theorem 3.88]. For this purpose, we can, analogously to the proof of Theorem 4.12, consider the local setting of $\Gamma \subset \partial \Omega$ with associated $y_{\beta}$ for $|\beta|=k$ where it suffices to prove that $u^{n}\left(y_{\beta}, \ldots, y_{\beta}\right)=u_{\beta}^{n} \rightarrow u_{\beta}=u\left(y_{\beta}, \ldots, y_{\beta}\right)$ in $L^{1}(\Gamma)$. Observe that for $0<t<t_{0}$, since $t \mapsto u_{\beta}\left(x-t y_{\beta}\right)$ is of bounded variation of almost every $x \in \Gamma$, the averaging

$$
\left(I_{t} u_{\beta}\right)(x)=\frac{1}{t} \int_{0}^{t} u_{\beta}\left(x-t y_{\beta}\right) \mathrm{d} t
$$

fulfills the elementary estimate

$$
\left|\gamma u_{\beta}(x)-\left(I_{t} u_{\beta}\right)(x)\right| \leq\left|u_{\beta}\left(P_{y_{\beta}}^{\perp} x\right)^{\prime}\right|(] x \cdot y_{\beta}-t, x \cdot y_{\beta}[) .
$$

Again, with Lemmas 4.9 and 4.11, it follows that

$$
\int_{\Gamma}\left|\gamma u_{\beta}(x)-\left(I_{t} u_{\beta}\right)(x)\right| \mathrm{d} \mathcal{H}^{d-1}(x) \leq C\left|\frac{\partial u_{\beta}}{\partial y_{\beta}}\right|\left(\Omega_{\beta}^{t}\right)
$$

where $\left.\Omega_{\beta}^{t}=\Gamma+\right]-t, 0\left[y_{\beta}\right.$. So, applying Lemma 4.11 once more yields

$$
\begin{aligned}
\int_{\Gamma} \mid \gamma u_{\beta}^{n}(x) & -\gamma u_{\beta}(x) \mid \mathrm{d} \mathcal{H}^{d-1}(x) \\
& \leq \int_{\Gamma}\left|\gamma u_{\beta}^{n}(x)-\left(I_{t} u_{\beta}^{n}\right)(x)-\gamma u_{\beta}(x)+\left(I_{t} u_{\beta}\right)(x)\right| \mathrm{d} \mathcal{H}^{d-1}(x)
\end{aligned}
$$




$$
\begin{aligned}
& +\int_{\Gamma}\left|I_{t}\left(u_{\beta}^{n}-u_{\beta}\right)(x)\right| \mathrm{d} \mathcal{H}^{d-1}(x) \\
\leq & \left.C\left(\left|\frac{\partial u_{\beta}}{\partial y_{\beta}}\right|\left(\Omega_{\beta}^{t}\right)+\left|\frac{\partial u_{\beta}^{n}}{\partial y_{\beta}}\right|\left(\Omega_{\beta}^{t}\right)+\frac{1}{t} \int_{\Omega_{\beta}^{t}}\left|u_{\beta}^{n}-u_{\beta}\right| \mathrm{d} x\right)\right) .
\end{aligned}
$$

Taking the limes superior implies, since $u_{\beta}^{n} \rightarrow u_{\beta}$ in $L^{1}(\Omega)$ and with the help of Lemma 4.9,

$$
\begin{aligned}
\limsup _{n \rightarrow \infty} \int_{\Gamma}\left|\gamma u_{\beta}^{n}-\gamma u_{\beta}\right| \mathrm{d} \mathcal{H}^{d-1} & \leq C\left(\left|\frac{\partial u_{\beta}}{\partial y_{\beta}}\right|\left(\Omega_{\beta}^{t}\right)+\limsup _{n \rightarrow \infty}\left|\frac{\partial u_{\beta}^{n}}{\partial y_{\beta}}\right|\left(\Omega_{\beta}^{t}\right)\right) \\
& \leq C\left(|\mathcal{E}(u)|\left(\Omega_{\beta}^{t}\right)+\limsup _{n \rightarrow \infty}\left|\mathcal{E}\left(u^{n}\right)\right|\left(\Omega_{\beta}^{t}\right)\right) \\
& \leq C|\mathcal{E}(u)|\left(\Omega \cap \overline{\Omega_{\beta}^{t}}\right)
\end{aligned}
$$

where the last estimate is a consequence of $\left|\mathcal{E}\left(u^{n}\right)\right|\left(\Omega_{\beta}^{t}\right) \leq\left|\mathcal{E}\left(u^{n}\right)\right|(\Omega)-\left|\mathcal{E}\left(u^{n}\right)\right|\left(\Omega \backslash \overline{\Omega_{\beta}^{t}}\right)$ and $|\mathcal{E}(u)|\left(\Omega \backslash \overline{\Omega_{\beta}^{t}}\right) \leq \liminf _{n \rightarrow \infty}\left|\mathcal{E}\left(u^{n}\right)\right|\left(\Omega \backslash \overline{\Omega_{\beta}^{t}}\right)$, confer [1, Proposition 1.62]. Letting $t \rightarrow 0$, we find that the right-hand side vanishes since the characteristic functions of $\Omega \cap \overline{\Omega_{\beta}^{t}}$ vanish pointwise in $\Omega$. Consequently, $u_{\beta}^{n} \rightarrow u_{\beta}$ in $L^{1}(\Gamma)$. The argumentation can be carried out for each $|\beta|=k$ and also be globalized, leading to $u^{n} \rightarrow u$ in $L^{1}\left(\partial \Omega, \operatorname{Sym}^{k}\left(\mathbb{R}^{d}\right)\right)$.

For the validity of the divergence theorem, first suppose that $u$ is continuously differentiable in $\Omega$. As before, we localize the problem to a boundary part $\Gamma$ for which we also associate suitable $y_{\beta}$ and $\eta_{\beta}$. Decomposing $v=\sum_{|\beta|=k} v_{\beta}\left(y_{\beta} \otimes \cdots \otimes y_{\beta}\right)$ where $v_{\beta}=v \cdot \eta_{\beta}$ and with the device of smooth partition of unity, it suffices to examine

$$
\int_{\Omega^{\prime} \cap \Omega} u_{\beta} \frac{\partial\left(\varphi v_{\beta}\right)}{\partial y_{\beta}} \mathrm{d} x
$$

where $\Omega^{\prime}=\bigcap_{|\beta|=k}(\Gamma+]-t_{0}, t_{0}\left[y_{\beta}\right)$ and $\varphi$ is a smooth cutoff function compactly supported in $\Omega^{\prime}$. For $t>0$, denote $\Gamma_{\beta}^{t}=\Gamma-t y_{\beta}$ and $\left.\Omega_{\beta}^{t}=\Gamma-\right] t, t_{0}\left[y_{\beta}\right.$. The usual integration by parts formula yields

$$
\int_{\Omega_{\beta}^{t}} u_{\beta} \frac{\partial\left(\varphi v_{\beta}\right)}{\partial y_{\beta}} \mathrm{d} x=\int_{\Gamma_{t}} u_{\beta}\left(v \cdot y_{\beta}\right) \varphi v_{\beta} \mathrm{d} \mathcal{H}^{d-1}(x)-\int_{\Omega_{\beta}^{t}} \frac{\partial u_{\beta}}{\partial y_{\beta}} \varphi v_{\beta} \mathrm{d} x .
$$

We like to pass to the limit as $t \rightarrow 0$. While the convergence of the volume terms is clear, observe, for the surface term, with the help of Lemma 4.11 and letting $\left.\left(\Omega_{\beta}^{t}\right)^{\prime}=\Gamma-\right] 0, t\left[y_{\beta}\right.$, that

$$
\begin{aligned}
\int_{\Gamma}\left|(\gamma u)_{\beta}(x)-u_{\beta}\left(x-t y_{\beta}\right)\right| \mathrm{d} \mathcal{H}^{d-1}(x) & \leq \int_{\Gamma} \int_{0}^{t}\left|\frac{\partial u_{\beta}}{\partial y_{\beta}}\left(x-t y_{\beta}\right)\right| \mathrm{d} t \mathrm{~d} \mathcal{H}^{d-1}(x) \\
& \leq C \int_{\left(\Omega_{\beta}^{t}\right)^{\prime}}|\mathcal{E}(u)| \mathrm{d} x
\end{aligned}
$$


which vanishes as $t \rightarrow 0$. Hence, we have

$$
\int_{\Omega} u_{\beta} \frac{\partial\left(\varphi v_{\beta}\right)}{\partial y_{\beta}} \mathrm{d} x=\int_{\Gamma}(\gamma u)_{\beta}\left(v \cdot y_{\beta}\right) \varphi v_{\beta} \mathrm{d} \mathcal{H}^{d-1}(x)-\int_{\Omega} \frac{\partial u_{\beta}}{\partial y_{\beta}} \varphi v_{\beta} \mathrm{d} x .
$$

Summing up over all $|\beta|=k$ and using that $\| \mid$ is the projection onto $\operatorname{Sym}^{k}\left(\mathbb{R}^{d}\right)$ in $\mathcal{T}^{k}\left(\mathbb{R}^{d}\right)$ yields

$$
\int_{\Omega} u \cdot \operatorname{div}(\varphi v) \mathrm{d} x=\int_{\Gamma} \|(\gamma u \otimes v) \cdot \varphi v \mathrm{~d} \mathcal{H}^{d-1}(x)-\int_{\Omega} \mathcal{E}(u) \cdot \varphi v \mathrm{~d} x .
$$

Eventually, summing up over the elements in the partition of unity establishes (4.9). For general $u \in \operatorname{BD}\left(\Omega, \operatorname{Sym}^{k}\left(\mathbb{R}^{d}\right)\right)$, we can approximate with a strictly converging sequence $\left\{u^{n}\right\}$ of continuously differentiable tensor fields. By the continuity of the trace operator, each integral in (4.9) converges, yielding the divergence theorem in the limit.

Corollary 4.15 For a bounded Lipschitz domain $\Omega$, the zero extension Eu of a $u \in$ $\mathrm{BD}\left(\Omega, \operatorname{Sym}^{k}\left(\mathbb{R}^{d}\right)\right)$ belongs to $\mathrm{BD}\left(\mathbb{R}^{d}, \operatorname{Sym}^{k}\left(\mathbb{R}^{d}\right)\right)$ with

$$
\mathcal{E}(E u)=\mathcal{E}(u)-\| \mid(\gamma u \otimes v) \mathcal{H}^{d-1}\llcorner\partial \Omega .
$$

The corresponding operator is continuous with respect to strict convergence and satisfies, for some $C>0$,

$$
\|E u\|_{1}+\|\mathcal{E}(E u)\|_{\mathcal{M}} \leq C\left(\|u\|_{1}+\|\mathcal{E}(u)\|_{\mathcal{M}}\right) .
$$

Proof Each compactly supported $v \in \mathcal{C}^{1}\left(\mathbb{R}^{d}, \operatorname{Sym}^{k+1}\left(\mathbb{R}^{d}\right)\right)$ is in particular in $v \in$ $\mathcal{C}^{1}\left(\bar{\Omega}, \operatorname{Sym}^{k+1}\left(\mathbb{R}^{d}\right)\right)$, hence

$$
\int_{\mathbb{R}^{d}} u \cdot \operatorname{div} v \mathrm{~d} x=\int_{\partial \Omega} \|(\gamma u \otimes v) \cdot v \mathrm{~d} \mathcal{H}^{d-1}(x)-\int_{\Omega} \mathcal{E}(u) \cdot v \mathrm{~d} x
$$

and since the right-hand side is the integral of $v$ with respect to a Radon measure, $E u \in$ $\operatorname{BD}\left(\mathbb{R}^{d}, \operatorname{Sym}^{k}\left(\mathbb{R}^{d}\right)\right)$ with the claimed formula for $\mathcal{E}(E u)$. Finally, the continuity as well as the estimate follow essentially by the respective statements for the trace operator of Theorems 4.12 and 4.14.

Theorem 4.16 Let $\Omega$ be a bounded Lipschitz domain. Then, there is a continuous injection $\operatorname{BD}\left(\Omega, \operatorname{Sym}^{k}\left(\mathbb{R}^{d}\right)\right) \hookrightarrow L^{d /(d-1)}\left(\Omega, \operatorname{Sym}^{k}\left(\mathbb{R}^{d}\right)\right)$.

Proof Note that we can assume $d \geq 2$ and that Theorem 4.8 already establishes the result for continuously differentiable $u \in \operatorname{BD}\left(\mathbb{R}^{d}, \operatorname{Sym}^{k}\left(\mathbb{R}^{d}\right)\right)$. For $u \in \operatorname{BD}\left(\Omega, \operatorname{Sym}^{k}\left(\mathbb{R}^{d}\right)\right)$, we can approximate, by applying Proposition 4.13 to a bounded domain $\Omega^{\prime}$ such that $\bar{\Omega} \subset \subset \Omega^{\prime}$, the zero extension $E u$ by some strictly converging sequence of continuously differentiable, compactly supported functions $\left\{u^{n}\right\}$. According to Theorem 4.8,

$$
\left\|u^{n}\right\|_{d /(d-1)} \leq C\left(\left\|u^{n}\right\|_{1}+\left\|\mathcal{E}\left(u^{n}\right)\right\|_{\mathcal{M}}\right)
$$

for each $n$. In $L^{1}\left(\Omega, \operatorname{Sym}^{k}\left(\mathbb{R}^{d}\right)\right)$, we have $u^{n} \rightarrow E u$ and as the $d /(d-1)$-norm is lower semi-continuous,

$$
\|u\|_{d /(d-1)}=\|E u\|_{d /(d-1)} \leq C\left(\|E u\|_{1}+\|\mathcal{E}(E u)\|_{\mathcal{M}}\right) \leq C\left(\|u\|_{1}+\|\mathcal{E}(u)\|_{\mathcal{M}}\right)
$$

taking moreover (4.10) into account. This establishes the continuous injection. 
Theorem 4.17 Let $\Omega$ be a bounded Lipschitz domain, $1 \leq p<d /(d-1)$ and $\left\{u^{n}\right\}$ be a bounded sequence in $\operatorname{BD}\left(\Omega, \operatorname{Sym}^{k}\left(\mathbb{R}^{d}\right)\right)$. Then, a subsequence $\left\{u^{n_{l}}\right\}$ converges, in $L^{p}\left(\Omega, \operatorname{Sym}^{k}\left(\mathbb{R}^{d}\right)\right)$, to a $u \in L^{p}\left(\Omega, \operatorname{Sym}^{k}\left(\mathbb{R}^{d}\right)\right)$.

Proof This proof is essentially based on [15, Theorem II.1.4]. We start with proving that the injection $\operatorname{BD}\left(\Omega, \operatorname{Sym}^{k}\left(\mathbb{R}^{d}\right)\right) \rightarrow L^{1}\left(\Omega, \operatorname{Sym}^{k}\left(\mathbb{R}^{d}\right)\right)$ is compact. For this purpose, fix an $\Omega^{\prime}$ for which $\bar{\Omega} \subset \subset \Omega^{\prime}$ and let $u \in \mathcal{C}_{\mathrm{c}}^{2}\left(\mathbb{R}^{d}, \operatorname{Sym}^{k}\left(\mathbb{R}^{d}\right)\right)$ with support in $\Omega^{\prime}$. Our aim is to prove the estimate

$$
\int_{\mathbb{R}^{d}}|u(\bar{x}+h)-u(\bar{x})| \mathrm{d} \bar{x} \leq C|h|^{s}\|\mathcal{E}(u)\|_{1}
$$

for some $s>0$ and all $h \in \mathbb{R}^{d},|h| \leq 1$ with a constant $C>0$ independent of $u$. Choose $\left\{y_{\beta}\right\}$ according to Lemma 4.5 (with $\bar{U}=S^{d-1}$, for instance) and set $\eta_{\beta}=y_{\beta} \otimes \cdots \otimes y_{\beta}$. Fix a multiindex $\beta$ with $|\beta|=k$. With Green's representation formula (3.15) as well as (4.9) (note that $\mathcal{E}\left(\Gamma_{\eta_{\beta}}^{k}\right) \in L_{\text {loc }}^{1}\left(\mathbb{R}^{d}, \operatorname{Sym}^{k+1}\left(\mathbb{R}^{d}\right)\right.$ ) by Lemma 3.9) we get for $\bar{x} \in \mathbb{R}^{d}$

$$
u(\bar{x}) \cdot \eta_{\beta}=\int_{\mathbb{R}^{d}} \Gamma_{\eta_{\beta}}^{k}(x-\bar{x}) \cdot \operatorname{div}(\mathcal{E}(u))(x) \mathrm{d} x=-\int_{\mathbb{R}^{d}} \mathcal{E}\left(\Gamma_{\eta_{\beta}}^{k}\right)(x-\bar{x}) \cdot \mathcal{E}(u)(x) \mathrm{d} x .
$$

Consequently, choosing some $0<s<1$ and applying (3.16), we can estimate for all $|h| \leq 1$

$$
\begin{aligned}
\int_{\Omega^{\prime}} \mid(u(\bar{x}+h) & -u(\bar{x})) \cdot \eta_{\beta} \mid \mathrm{d} \bar{x} \\
& \leq \int_{\Omega^{\prime}-\Omega^{\prime}}\left|\mathcal{E}\left(\Gamma_{\eta_{\beta}}^{k}\right)(\cdot-h)-\mathcal{E}\left(\Gamma_{\eta_{\beta}}^{k}\right)\right| \mathrm{d} \bar{x} \int_{\Omega^{\prime}}|\mathcal{E}(u)| \mathrm{d} x \\
& \leq C|h|^{s} \int_{\Omega^{\prime}-\Omega^{\prime}}|\bar{x}-h|^{1-d-s}+|\bar{x}|^{1-d-s} \mathrm{~d} \bar{x} \int_{\Omega^{\prime}}|\mathcal{E}(u)| \mathrm{d} x \\
& \leq C|h|^{s}\|\mathcal{E}(u)\|_{1} .
\end{aligned}
$$

By the norm equivalence established in Lemma 4.5, one arrives at

$$
\begin{aligned}
\int_{\mathbb{R}^{d}}|u(\bar{x}+h)-u(\bar{x})| \mathrm{d} x & \leq C \int_{\Omega^{\prime}} \sum_{|\beta|=k}\left|(u(\bar{x}+h)-u(\bar{x})) \cdot \eta_{\beta}\right| \mathrm{d} \bar{x} \\
& \leq C|h|^{s}\|\mathcal{E}(u)\|_{1} .
\end{aligned}
$$

Now, let $u \in \operatorname{BD}\left(\Omega, \operatorname{Sym}^{k}\left(\mathbb{R}^{d}\right)\right)$ be arbitrary. According to Corollary 4.15 , the zero extension $E u$ satisfies $E u \in \operatorname{BD}\left(\Omega^{\prime}, \operatorname{Sym}^{k}\left(\mathbb{R}^{d}\right)\right)$. Obviously, $E u$ has compact support in $\Omega^{\prime}$, so by Proposition 4.13 , there is a sequence $\left\{v^{n}\right\}$ in $\mathcal{C}_{\mathrm{c}}^{\infty}\left(\Omega^{\prime}, \operatorname{Sym}^{k}\left(\mathbb{R}^{d}\right)\right)$ for which $v^{n} \rightarrow E u$ in $L^{1}\left(\Omega, \operatorname{Sym}^{k}\left(\mathbb{R}^{d}\right)\right)$ as well as $\left\|\mathcal{E}\left(v^{n}\right)\right\|_{1} \rightarrow\|\mathcal{E}(E u)\|_{\mathcal{M}}$ as $n \rightarrow \infty$. Plugging this into (4.11) and passing to the limit yields

$$
\int_{\mathbb{R}^{d}}|E u(\bar{x}+h)-E u(\bar{x})| \mathrm{d} \bar{x} \leq C|h|^{s}\|\mathcal{E}(E u)\|_{\mathcal{M}} \leq C|h|^{s}\left(\|u\|_{1}+\|\mathcal{E}(u)\|_{\mathcal{M}}\right),
$$

the latter by (4.10). Therefore, for a bounded sequence in $\operatorname{BD}\left(\Omega, \operatorname{Sym}^{k}\left(\mathbb{R}^{d}\right)\right)$, the $\left\{E u^{n}\right\}$ are relatively compact [7, Theorem IV.8.21] and therefore, there exists a $u \in L^{1}\left(\mathbb{R}^{d}, \operatorname{Sym}^{k}\left(\mathbb{R}^{d}\right)\right)$ and a subsequence $\left\{E u^{n_{l}}\right\}$ with $E u^{n_{l}} \rightarrow u$. It is immediate that $\left.u^{n_{l}} \rightarrow u\right|_{\Omega}$ in 
$L^{1}\left(\Omega, \operatorname{Sym}^{k}\left(\mathbb{R}^{d}\right)\right)$ which establishes the compact embedding $\operatorname{BD}\left(\Omega, \operatorname{Sym}^{k}\left(\mathbb{R}^{d}\right)\right) \hookrightarrow$ $L^{1}\left(\Omega, \operatorname{Sym}^{k}\left(\mathbb{R}^{d}\right)\right)$.

The general case $1 \leq p<d /(d-1)$ then follows by taking the boundedness of $\left\{u^{n}\right\}$ in $L^{d /(d-1)}\left(\Omega, \operatorname{Sym}^{k}\left(\mathbb{R}^{d}\right)\right)$ into account, compare Theorem 4.16. Let, without loss of generality, $u^{n} \rightarrow u$ in $L^{1}\left(\Omega, \operatorname{Sym}^{k}\left(\mathbb{R}^{d}\right)\right)$ and $\left\{u^{n}\right\}$ be bounded in $L^{d /(d-1)}\left(\Omega, \operatorname{Sym}^{k}\left(\mathbb{R}^{d}\right)\right)$ with bound $M$. First note that, for $\delta>0$, we can write, with $p_{1}=d-(d-1) p$ and $p_{2}=(p-1) d$ the $p$ th power of a $t \geq 0$ as $t^{p}=\left(t^{p_{1}} / \delta\right)\left(t^{p_{2}} \delta\right)$ and apply Young's inequality with $q_{1}=(d-(d-1) p)^{-1}$ and $q_{2}=(p-1)^{-1}(d-1)^{-1}$ to obtain, for each $t \geq 0$ the elementary estimate

$$
t^{p} \leq \frac{t^{p_{1} q_{1}}}{\delta^{q_{1}} q_{1}}+\delta^{q_{2}} \frac{t^{p_{2} q_{2}}}{q_{2}}=\frac{t}{\delta^{q_{1}} q_{1}}+\delta^{q_{2}} \frac{t^{d /(d-1)}}{q_{2}}
$$

which leads to

$$
\int_{\Omega}\left|u^{n}-u\right|^{p} \mathrm{~d} x \leq\left(\delta^{q_{1}} q_{1}\right)^{-1} \int_{\Omega}\left|u^{n}-u\right| \mathrm{d} x+\frac{\delta^{q_{2}}}{q_{2}} \int_{\Omega}\left|u^{n}-u\right|^{d /(d-1)} \mathrm{d} x .
$$

Now, let a $\varepsilon>0$ be given. Choose $\delta>0$ such that $M^{d /(d-1)} \delta^{q_{2}} / q_{2}<\varepsilon^{p} / 2$ and $n_{0}$ such that $\left\|u^{n}-u\right\|_{1}<\delta^{q_{1}} q_{1} \varepsilon^{p} / 2$ for each $n \geq n_{0}$. With the above estimate, we then have

$$
\int_{\Omega}\left|u^{n}-u\right|^{p} \mathrm{~d} x \leq \frac{\varepsilon^{p}}{2}+\frac{\varepsilon^{p}}{2}=\varepsilon^{p}
$$

which means that $u^{n} \rightarrow u$ also in $L^{p}\left(\Omega, \operatorname{Sym}^{k}\left(\mathbb{R}^{d}\right)\right)$.

Our compactness result enables us to obtain an inequality of Sobolev-Korn type. As a preparation, we need to define projectors on the kernel of the symmetrized derivative for which a characterization has been obtained in Proposition 3.3.

Definition 4.18 For a bounded domain $\Omega$ and $k \geq 0$, denote by

$$
\begin{aligned}
\mathcal{R}_{k}=\{u: \Omega & \rightarrow \operatorname{Sym}^{k}\left(\mathbb{R}^{d}\right) \mid \forall x \in \Omega: \\
& u(x)=\sum_{l=0}^{k} A_{l}(\underbrace{x, \ldots, x}_{l \text { times }}, \cdot), A_{l} \in \operatorname{Sym}^{l}\left(\mathbb{R}^{d}\right) \otimes \operatorname{Sym}^{k}\left(\mathbb{R}^{d}\right), \\
& \forall x_{1}, \ldots, x_{l-1} \in \mathbb{R}^{d}, y_{1}, \ldots, y_{k+1} \in \mathbb{R}^{d}: \\
& \left.\sum_{m=1}^{k+1} A_{l}\left(x_{1}, \ldots, x_{l-1}, y_{m}, \ldots, y_{k+1}, y_{1}, \ldots, y_{m-1}\right)=0\right\}
\end{aligned}
$$

the space of infinitesimal rigid body displacements of order $k$.

Theorem 4.19 Let $\Omega$ be a bounded Lipschitz domain, $k \geq 1$ and $R: L^{1}\left(\Omega, \operatorname{Sym}^{k}\left(\mathbb{R}^{d}\right)\right) \rightarrow$ $\mathcal{R}_{k}$ a linear projection. Then, there exists $a C>0$ such that for each $u \in \operatorname{BD}\left(\Omega, \operatorname{Sym}^{k}\left(\mathbb{R}^{d}\right)\right)$, the estimate

$$
\|u-R u\|_{1} \leq C\|\mathcal{E}(u)\|_{\mathcal{M}}
$$

is satisfied. 
Proof First note that we can restrict ourselves to proving that, for some $C>0$,

$$
\|u-R u\|_{1} \leq C\left(\|\mathcal{E}(u)\|_{\mathcal{M}}+1\right)
$$

for each $u \in \operatorname{BD}\left(\Omega, \operatorname{Sym}^{k}\left(\mathbb{R}^{d}\right)\right)$ : Replacing $u$ by $t u$ with $t>0$ and dividing the above estimate by $t$ yields (4.12) as $t \rightarrow \infty$.

Thus, suppose the above statement is wrong, then there would be a sequence $\left\{u^{n}\right\}$ in $\operatorname{BD}\left(\Omega, \operatorname{Sym}^{k}\left(\mathbb{R}^{d}\right)\right)$ with

$$
\left\|\mathcal{E}\left(u^{n}\right)\right\|_{\mathcal{M}}+1 \leq \frac{1}{n}\left\|u^{n}-R u^{n}\right\|_{1}
$$

for each $n$. Thus, the sequence defined by $v^{n}=\left(u^{n}-R u^{n}\right) /\left\|u^{n}-R u^{n}\right\|_{1}$ obeys $\left\|v^{n}\right\|_{1}=1$, $\left\|\mathcal{E}\left(v^{n}\right)\right\|_{\mathcal{M}}=\left\|u^{n}-R u^{n}\right\|_{1}^{-1}\left\|\mathcal{E}\left(u^{n}\right)\right\|_{\mathcal{M}}$ and, consequently,

$$
\left\|\mathcal{E}\left(v^{n}\right)\right\|_{\mathcal{M}} \leq\left\|\mathcal{E}\left(v^{n}\right)\right\|_{\mathcal{M}}+\left\|u^{n}-R u^{n}\right\|_{1}^{-1} \leq \frac{1}{n} .
$$

It follows that $\left\{v^{n}\right\}$ is bounded in $\operatorname{BD}\left(\Omega, \operatorname{Sym}^{k}\left(\mathbb{R}^{d}\right)\right)$ with $\mathcal{E}\left(v^{n}\right) \rightarrow 0$. Since the embedding into $L^{1}\left(\Omega, \operatorname{Sym}^{k}\left(\mathbb{R}^{d}\right)\right.$ ) is compact (see Theorem 4.17), there is a subsequence $\left\{v^{n}\right\}$ (which is not relabeled) such that $v^{n} \rightarrow v$ in $L^{1}\left(\Omega, \operatorname{Sym}^{k}\left(\mathbb{R}^{d}\right)\right)$ and $\mathcal{E}\left(v^{n}\right) \rightarrow 0$ in $\mathcal{M}\left(\Omega, \operatorname{Sym}^{k+1}\left(\mathbb{R}^{d}\right)\right)$. By the closedness of the symmetrized derivative (see Remark 4.3), it follows that $\mathcal{E}(v)=0$, or, equivalently, $v \in \mathcal{R}_{k}$ (see Proposition 3.3).

On the other hand, for each $n$, we have $v^{n} \in \operatorname{rg}(I-R) \subset L^{1}\left(\Omega, \operatorname{Sym}^{k}\left(\mathbb{R}^{d}\right)\right)$ and since this subspace is closed, $v \in \operatorname{rg}(I-R)$. But $L^{1}\left(\Omega, \operatorname{Sym}^{k}\left(\mathbb{R}^{d}\right)\right)=\mathcal{R}_{k} \oplus \operatorname{rg}(I-R)$, so with the above follows $v=0$. Due to the strong convergence, $\left\|v^{n}\right\|_{1} \rightarrow 0$ as $n \rightarrow \infty$, which is obviously a contradiction to $\left\|v^{n}\right\|_{1}=1$ for each $n$. Thus, the estimate (4.13) has to hold and consequently (4.12).

Finally, the Sobolev-Korn inequality in $L^{d /(d-1)}\left(\Omega, \operatorname{Sym}^{k}\left(\mathbb{R}^{d}\right)\right)$ comes as a corollary.

Corollary 4.20 For $\Omega \subset \mathbb{R}^{d}$ a bounded Lipschitz domain, $d \geq 2, k \geq 1$ and $R$ : $L^{d /(d-1)}\left(\Omega, \operatorname{Sym}^{k}\left(\mathbb{R}^{d}\right)\right) \rightarrow \mathcal{R}_{k}$ a linear projection, it holds that

$$
\|u-R u\|_{d /(d-1)} \leq C\|\mathcal{E}(u)\|_{\mathcal{M}}
$$

for all $u \in \operatorname{BD}\left(\Omega, \operatorname{Sym}^{k}\left(\mathbb{R}^{d}\right)\right)$ and some $C>0$ independent of $u$.

Proof This easily follows from combining Theorem 4.16 with Theorem 4.19:

$$
\|u-R u\|_{d /(d-1)} \leq C\left(\|u-R u\|_{1}+\|\mathcal{E}(u)\|_{\mathcal{M}}\right) \leq C\|\mathcal{E}(u)\|_{1} .
$$

\section{Appendix A: Additional results}

The following two lemmas are concerned with the operators $\xi \otimes_{L} \xi$ which are used to obtain the fundamental solutions of $\operatorname{div} \mathcal{E}$ in Sect. 3.

Lemma A.1 Let $k \geq 1, \xi \in \mathbb{R}^{d}$ with $|\xi|=1$ and for each $L \subset\{1, \ldots, k\}$ the operator $\left(\xi \otimes_{L} \xi\right) \in \mathcal{L}\left(\mathcal{T}^{k}\left(\mathbb{R}^{d}\right), \mathcal{T}^{k}\left(\mathbb{R}^{d}\right)\right)$ be defined by

$$
\left(\left(\xi \otimes_{L} \xi\right) v\right)\left(a_{1}, \ldots, a_{k}\right)=\left(\prod_{l \in L} a_{l} \cdot \xi\right) \sum_{p: L \rightarrow\{1, \ldots, d\}} \xi_{p} v\left(a_{p}\right)
$$


where, with $\mathcal{D}(p)=L$,

$$
\xi_{p}=\prod_{l \in \mathcal{D}(p)} \xi_{p_{l}}, \quad\left(a_{p}\right)_{i}= \begin{cases}e_{p_{i}} & \text { if } i \in \mathcal{D}(p), \\ a_{i} & \text { else. }\end{cases}
$$

Then, for $L, M \subset\{1, \ldots, k\}$ it holds that

$$
\left(\xi \otimes_{L} \xi\right)\left(\xi \otimes_{M} \xi\right)=\left(\xi \otimes_{L \cup M} \xi\right) .
$$

Proof First observe that for each $p: L \rightarrow\{1, \ldots, d\}$ and $v \in \mathcal{T}^{k}\left(\mathbb{R}^{d}\right)$ it holds that

$$
\left(\left(\xi \otimes_{M} \xi\right) v\right)\left(a_{p}\right)=\left(\prod_{m \in M \backslash L} a_{m} \cdot \xi\right) \xi_{\left.p\right|_{L \cap M}} \sum_{q: M \rightarrow\{1, \ldots, d\}} \xi_{q} v\left(a_{p, q}\right)
$$

with

$$
\left(a_{p, q}\right)_{i}= \begin{cases}e_{q_{i}} & \text { if } i \in M, \\ e_{p_{i}} & \text { if } i \in L \backslash M, \\ a_{i} & \text { else. }\end{cases}
$$

Also note that $\xi_{p}=\xi_{\left.p\right|_{L \backslash M}} \xi_{\left.p\right|_{L \cap M}}$ as well as $a_{p, q}=a_{\left.p\right|_{L \backslash M}, q}$. Together with the assumption $|\xi|^{2}=1$, one deduces

$$
\begin{aligned}
\left(\left(\xi \otimes_{L} \xi\right)\right. & \left.\left(\xi \otimes_{M} \xi\right) v\right)\left(a_{1}, \ldots, a_{k}\right) \\
& =\left(\prod_{l \in L} a_{l} \cdot \xi\right) \sum_{p: L \rightarrow\{1, \ldots, d\}} \xi_{p}\left(\left(\xi \otimes_{M} \xi\right) v\right)\left(a_{p}\right) \\
& =\left(\prod_{l \in L} a_{l} \cdot \xi\right)\left(\prod_{m \in M \backslash L} a_{m} \cdot \xi\right) \sum_{\substack{p: L \rightarrow\{1, \ldots, d\} \\
q: M \rightarrow\{1, \ldots, d\}}} \xi_{p} \xi_{\left.p\right|_{L \cap M} \xi_{q} v\left(a_{p, q}\right)} \\
& =\left(\prod_{\mu \in L \cup M} a_{\mu} \cdot \xi \sum_{\substack{p: L \rightarrow\{1, \ldots, d\} \\
q: M \rightarrow\{1, \ldots, d\}}} \xi_{\left.p\right|_{L \cap M}}^{2} \xi_{\left.p\right|_{L \backslash M}} \xi_{q} v\left(a_{p, q}\right)\right. \\
& \left.=\left(\prod_{\mu \in L \cup M} a_{\mu} \cdot \xi\right)_{\substack{p: L \backslash M \rightarrow\{1, \ldots, d\} \\
q: M \rightarrow\{1, \ldots, d\}}} \sum_{\sigma: L \cap M \rightarrow\{1, \ldots, d\}} \xi_{\sigma}^{2}\right) \xi_{p} \xi_{q} v\left(a_{p, q}\right) \\
& =\left(\prod_{\mu \in L \cup M} a_{\mu} \cdot \xi \sum_{\substack{\pi: L \cup M \rightarrow\{1, \ldots, d\} \\
\left(a_{1}, \ldots, a_{k}\right) .}} \xi_{\pi} v\left(a_{\pi}\right)\right. \\
& =\left(\left(\xi \otimes_{L \cup M} \xi\right) v\right)
\end{aligned}
$$

Lemma A.2 Let $k \geq 1,0 \leq j \leq k$ and $v: \mathbb{R}^{d} \rightarrow \operatorname{Sym}^{k}\left(\mathbb{R}^{d}\right)$ a Schwartzfunction. Then, for all $\xi \in \mathbb{R}^{d}$,

$$
\mathcal{F}\left(\mathcal{E}^{j}\left(\operatorname{div}^{j} v\right)\right)(\xi)=(-1)^{j}\left(\begin{array}{l}
k \\
j
\end{array}\right)^{-1} \sum_{|L|=j}\left(\xi \otimes_{L} \xi\right) \mathcal{F} v(\xi)
$$

with $\xi \otimes_{L} \xi$ according to (A.1). 
Proof One can verify, without greater effort, that

$$
\begin{array}{r}
\left(\mathcal{E}^{j}\left(\operatorname{div}^{j} v\right)\right)(x)\left(a_{1}, \ldots, a_{k}\right)=\frac{1}{k !} \sum_{\pi \in S_{k}} \sum_{m_{1}, \ldots, m_{j}=1}^{d}\left(\prod_{\mu=1}^{j} a_{\pi_{\mu}, m_{\mu}}\right) \\
\times \sum_{i_{1}, \ldots, i_{j}=1}^{d} \frac{\partial^{2 j} v}{\partial x_{m_{1}} \cdots \partial x_{m_{j}} \partial x_{i_{1}} \cdots \partial x_{i_{j}}}(x)\left(a_{\pi_{j+1}}, \ldots, a_{\pi_{k}}, e_{i_{1}}, \ldots, e_{i_{j}}\right) .
\end{array}
$$

Taking the Fourier transform at a point $\xi$ therefore yields

$$
\begin{aligned}
& \mathcal{F}\left(\mathcal{E}^{j}\left(\operatorname{div}^{j} v\right)\right)(\xi)\left(a_{1}, \ldots, a_{k}\right)=\frac{(-1)^{j}}{k !} \sum_{\pi \in S_{k}} \sum_{m_{1}, \ldots, m_{j}=1}^{d}\left(\prod_{\mu=1}^{j} a_{\pi_{\mu}, m_{\mu}}\right) \\
& \times \sum_{i_{1}, \ldots, i_{j}=1}^{d}\left(\prod_{\mu=1}^{j} \xi_{m_{\mu}}\right)\left(\prod_{v=1}^{j} \xi_{i_{v}}\right)(\mathcal{F} v)(\xi)\left(a_{\pi_{j+1}}, \ldots, a_{\pi_{k}}, e_{i_{1}}, \ldots, e_{i_{j}}\right) .
\end{aligned}
$$

Now, associate, for each $\pi \in S_{k}$, the set $L=\left\{\pi_{1}, \ldots, \pi_{j}\right\}$ and rewrite

$$
\sum_{m_{1}, \ldots, m_{j}=1}^{d}\left(\prod_{\mu=1}^{j} a_{\pi_{\mu}, m_{\mu}}\right)\left(\prod_{\mu=1}^{j} \xi_{m_{\mu}}\right)=\left(\prod_{\mu=1}^{j} a_{\pi_{\mu}} \cdot \xi\right)=\left(\prod_{l \in L} a_{l} \cdot \xi\right)
$$

which is obviously invariant if one leaves $L$ fixed and changes $\pi \in S_{k}$ such that still $\left\{\pi_{1}, \ldots, \pi_{j}\right\}=L$. Moreover, all $1 \leq i_{1}, \ldots, i_{j} \leq d$ correspond one-to-one to all $p: L \rightarrow\{1, \ldots, d\}$ which allows to rewrite, due to the symmetry of $(\mathcal{F} v)(\xi)$, the expression

$$
\left(\prod_{v=1}^{j} \xi_{i_{v}}\right)(\mathcal{F} v)(\xi)\left(a_{\pi_{j+1}}, \ldots, a_{\pi_{k}}, e_{i_{1}}, \ldots, e_{i_{j}}\right)=\xi_{p} \mathcal{F} v(\xi)\left(a_{p}\right)
$$

where $p: L \rightarrow\{1, \ldots, d\}$ is uniquely determined. The expression on the right-hand side is invariant for all $\pi \in S_{k}$ with $\pi_{j+1}, \ldots, \pi_{k} \notin L$ and $L$ fixed. Exploiting these observations in (A.3) leads to

$$
\begin{aligned}
\mathcal{F}\left(\mathcal{E}^{j}\left(\operatorname{div}^{j} v\right)\right)(\xi)\left(a_{1}, \ldots, a_{k}\right) \\
=\frac{(-1)^{j}}{k !} \sum_{|L|=j} \sum_{\substack{\pi \in S_{k} \\
\pi_{1}, \ldots, \pi_{j} \in L \\
\pi_{j+1}, \ldots, \pi_{k} \notin L}}\left(\prod_{l \in L} a_{l} \cdot \xi\right) \sum_{p: L \rightarrow\{1, \ldots, d\}} \xi_{p} \mathcal{F} v(\xi)\left(a_{p}\right) \\
=\frac{(-1)^{j}}{k !} \sum_{|L|=j} j !(k-j) !\left(\xi \otimes_{L} \xi\right) \mathcal{F} v(\xi)\left(a_{1}, \ldots, a_{k}\right) \\
=\left((-1)^{j}\left(\begin{array}{c}
k \\
j
\end{array}\right)^{-1} \sum_{|L|=j}\left(\xi \otimes_{L} \xi\right) \mathcal{F} v(\xi)\right)\left(a_{1}, \ldots, a_{k}\right),
\end{aligned}
$$

hence, (A.2) is proved. 
Next, we like to collect, for the reader's convenience, some transformation rules for (symmetric) $k$-tensor fields as well as its (symmetrized) gradient. First, recall that for a linear transformation $O \in \mathbb{R}^{d \times d}$, the right-multiplication

$$
(\xi O)\left(a_{1}, \ldots, a_{k}\right)=\xi\left(O a_{1}, \ldots, O a_{k}\right)
$$

also gives a symmetric $k$-linear mapping for which one can see that with $p=\sigma^{-1}(\beta)$ (confer Sect. 2), the coefficients obey

$$
(\xi O)_{\beta}=\sum_{q \in\{1, \ldots, d\}^{k}} \prod_{j=1}^{k} o_{q_{j} p_{j}} \xi_{\sigma(q)} .
$$

Let us also note that right-multiplication with orthonormal $O \in \mathbb{R}^{d \times d}$ commutes with the trace of a $\xi \in \mathcal{T}^{k}\left(\mathbb{R}^{d}\right)$ with $k \geq 2$ :

$$
\begin{aligned}
\operatorname{tr}(\xi O)\left(a_{1}, \ldots, a_{k-2}\right) & =\sum_{i, j, j^{\prime}=1}^{d} o_{j i} o_{j^{\prime} i} \xi\left(e_{j}, O a_{1}, \ldots, O a_{k-2}, e_{j^{\prime}}\right) \\
& =\sum_{i=1}^{d} \xi\left(e_{i}, O a_{1}, \ldots, O a_{k-2}, e_{i}\right) \\
& =(\operatorname{tr}(\xi) O)\left(a_{1}, \ldots, a_{k-2}\right) .
\end{aligned}
$$

Hence, right-multiplication respects orthonormality:

Lemma A.3 If $O \in \mathbb{R}^{d \times d}$ is orthonormal, the operation $\xi \mapsto \xi O$ is an orthonormal transformation mapping $\operatorname{Sym}^{k}\left(\mathbb{R}^{d}\right) \rightarrow \operatorname{Sym}^{k}\left(\mathbb{R}^{d}\right)$.

Proof Applying (A.5) $k$ times to $(\xi O \otimes \eta O)=(\xi \otimes \eta) O$ yields

$$
\xi O \cdot \eta O=\operatorname{tr}^{k}((\xi \otimes \eta) O)=\operatorname{tr}^{k}(\xi \otimes \eta) O=\xi \cdot \eta .
$$

It turns out that the right-multiplication is moreover an appropriate notion for describing the transformation behavior under linear coordinate changes.

Lemma A.4 Let $O \in \mathbb{R}^{d \times d}$ and $\xi: \Omega \rightarrow \mathcal{T}^{k}\left(\mathbb{R}^{d}\right)$ an l times continuously differentiable mapping. Then:

$$
\nabla^{l} \otimes((\xi \circ O) O)=\left(\left(\nabla^{l} \otimes \xi\right) \circ O\right) O
$$

as well as

$$
\mathcal{E}^{l}((u \circ O) O)=\left(\mathcal{E}^{l}(u) \circ O\right) O .
$$

Proof Set $\eta(x)=\xi(O x) O$ and compute

$$
\begin{aligned}
\left(\nabla^{l} \otimes \eta\right)(x)\left(a_{1}, \ldots, a_{k+l}\right) & =\left(\mathrm{D}^{l}(\xi \circ O)(x)\left(a_{1}, \ldots, a_{k}\right)\right)\left(O a_{k+1}, \ldots, O a_{k+l}\right) \\
& =\left(\mathrm{D}^{l} \xi(O x)\left(O a_{1}, \ldots, O a_{k}\right)\right)\left(O a_{k+1}, \ldots, O a_{k+l}\right) \\
& =\left(\left(\nabla^{l} \otimes \xi\right) O\right)(O x)\left(a_{1}, \ldots, a_{k+l}\right) .
\end{aligned}
$$

Symmetrizing the left- and right-hand side furthermore yields $\mathcal{E}^{l}(\eta)(x)=\mathcal{E}^{l}(\xi)(O x) O$. 


\section{References}

1. Ambrosio, L., Fusco, N., Pallara, D.: Functions of Bounded Variation and Free Discontinuity Problems. Oxford University Press, Oxford (2000)

2. Anzellotti, G., Giaquinta, M.: Existence of the displacements field for an elasto-plastic body subject to Hencky's law and Von Mises yield condition. Manuscripta Math. 32, 101-136 (1980). doi:10.1007/ BF01298185

3. Biggs, N.: Algebraic Graph Theory, 2nd edn. Cambridge University Press, Cambridge (1993)

4. Bredies, K., Kunisch, K., Pock, T.: Total generalized variation. SIAM J. Imaging Sci. 3(3), 492-526 (2010)

5. Bredies, K., Valkonen, T.: Inverse problems with second-order total generalized variation constraints. In: Proceedings of the 9th International Conference on Sampling Theory and Applications (2011)

6. Chang, C.S., Gao, J.: Second-gradient constitutive theory for granular material with random packing structure. Int. J. Solids Struct. 32(16), 2279-2293 (1995)

7. Dunford, N.J., Schwartz, J.T.: Linear Operators. Part I: General Theory, Pure and Applied Mathematics. Vol. 7, Wiley, New York (1958)

8. Evans, L.C., Gariepy, R.F.: Measure Theory and Fine Properties of Functions. CRC Press, UK (1992)

9. Gargiulo, G., Zappale, E.: A lower semicontinuity result in SBD. J. Convex Anal. 15(1), 191-200 (2008)

10. Godsil, C., Royle, G.: Algebraic Graph Theory. Springer, New York (2001)

11. Kikuchi, N., Oden, J.T.: Contact Problems in Elasticity: A Study of Variational Inequalities and Finite Element Methods, SIAM Studies in Applied Mathematics. Vol. 8, Society for Industrial and Applied Mathematics (SIAM), Philadelphia (1988)

12. Schwartz, L.: Theorie des Distributions, Publications de l'institut de Mathematique de l'universite de Strasbourg. Vol. 10, Hermann, Paris (1966)

13. Strauss, M.J.: Variations of Korn's and Sobolev's equalities. In: Partial Differential Equations. (Proceedings of Symposia in Pure Mathematics, vol. XXIII, University of California, Berkeley 1971), pp. 207-214. American Mathematical Society, Providence (1973)

14. Suiker, A.S.J., Chang, C.S.: Application of higher-order tensor theory for formulating enhanced continuum models. Acta Mech. 142, 223-234 (2000). doi:10.1007/BF01190020

15. Temam, R.: Mathematical Problems in Plasticity. Bordas, Paris (1985)

16. Temam, R., Strang, G.: Functions of bounded deformation. Arch. Ration. Mech. Anal. 75(1), 7-21 (1980) 